\title{
L'OPERA DEI 'CRISTALLERI'. CRISTALLI DI ROCCA, DIASPRI, OREFICERIE E RELIQUIE A VENEZIA (SECC. XIII-XIV)
}

MICHELA AGAZZI

UDC: $748(450.341) " 12 / 13 "$

Original scientific paper

Manuscript received: 11. 01. 2016.

Revised manuscript accepted: 10. 02. 2016.

DOI: 10.1484/J.HAM.5.111338

\author{
M. Agazzi \\ Università Ca' Foscari, Venezia \\ Dorsoduro 3246 \\ 30123 Venezia ITALIA \\ e-mail : agazzi@unive.it
}

The $13^{\text {th }}$ century saw the rise of the multi-material art of the cristalleri, who, using imported rock crystal, created objects from cut or baccellato blocks, or applied thin sheets of crystal to miniatures (creating pseudo-enamels) or to embossed foils, mounted together with inserts of red jasper or with filigree. The precious objects thus created were both secular (including precious chess-sets) and religious (especially crosses and reliquaries). At the end of the $13^{\text {th }}$ and throughout the $14^{\text {th }}$ century the technique of translucent enamel was developed. No object with miniatures has survived in Venice, where only the Treasure of St Mark's includes items connected with this specific Venetian art. It was thus principally designed for export, a product of the artistic (but also commercial) creativity of Venice, bringing together the fruits of the loot from Constantinople (relics) and imported minerals, and creating liturgical furnishings and luxury secular articles for a market that extended even beyond the confines of Europe.

Keywords: Venice, Cristalleri, crystal rock, goldsmith, reliquiaries, liturgical furniture, game boards, luxury products

Il corpus riferibile ai cristalleri veneziani medievali è stato ampiamente studiato e catalogato, ${ }^{1}$ esaminato sotto i diversi aspetti che caratterizzano gli artefatti preziosi di quell'arte, connotata dal polimaterismo e dalla associazione di diverse tecniche. Si vuole proporne qui una riconsiderazione sotto la prospettiva del tema del convegno, considerando il ruolo chiave di Venezia città- porto funzionante a scala europea e mediterranea nei secoli XIII-XIV.

L'arte dei cristalleri è normata a Venezia nel $1284,{ }^{2}$ ma la regolamentazione ovviamente segue l'avvio della attività artigianale , sorta evidentemente come una specializzazione dell'oreficeria (il capitolare degli oresi dava indicazioni anche per l'uso dei cristalli) ${ }^{3}$ e dimostra una prassi consolidata nell'utilizzo del cristallo di rocca (quarzo ialino) che possiamo far risalire a parecchi decenni prima. La nascita di questi oggetti - principalmente liturgici - trova sicuramente le sue origini fin dalla prima metà di quel secolo, nella fase di dominazione latina dell'ex impero bizantino, grazie al confronto diretto con i materiali ricchissimi immessi nel Tesoro di San Marco e nell'intera città. ${ }^{4}$ Alcuni pezzi riconducibili a quelle maestranze sono conservati proprio nella collezione marciana: lì sono confluiti da Costantinopoli importanti vasi e brocchette, rimontati nel Duecento con integrazioni orafe in gran parte caratterizzate dalla filigrana. ${ }^{5}$ (Fig. 1) Gli orafi veneziani coinvolti dai Procuratori di San Marco nell'ammodernamento e integrazione di cristalli antichi, fatimiti e bizantini si sono misurati quindi con oggetti di straordinario e riconosciuto valore, attribuendo loro anche nuove funzioni, come è il caso della cd Grotta della Vergine dove una edicola in cristallo di rocca, rovesciata, è diventata lo sfondo - che dobbiamo immaginare sfavillante alla luce delle candele - di una madonnina bronzea veneziana poggiata su

${ }^{1}$ Il corpus completo della produzione medievale in cristallo (non solo veneziana) è stato pubblicato da H. HAHNLOSER- S. BRUGGER KOCH , Corpus der Hartsteinschliffe des 12.-15. Jahrhunderts, Berlin 1985, mentre R.Degen ha catalogato specificatamente la produzione veneziana con miniature sotto cristallo (R. DEGEN, Venetianische Zimelien mit miniaturen unter berkristall des 13 und 14 Jahrunderts, Munster 2003); su questo aspetto particolare vedi inoltre: P. TOESCA, Un capolavoro dell'oreficeria veneziana della fine del Dugento, in Arte Veneta, 1951, pp. 15 -20; V. LAZAREV, Saggi sulla pittura veneziana dei secc. XII - XIV. La maniera greca e il problema della scuola cretese, in Arte Veneta, 1965, pp. 17-31; A. NEFF, Miniatori e arte dei cristalleri a Venezia nella seconda metà del Duecento, in Arte Veneta ,44, 1994, pp. 7-19; M. COLLARETA, Il cristallo nella liturgia religiosa e civile con qualche osservazione sulle croci veneziane in cristallo di rocca, in B. ZANETTIN (a cura di ), Cristalli e gemme, realtà fisica e immaginario, simbologia, tecniche e arte, Venezia 2003 , pp. 495-512; L. CASELLI, L'ornamento dei santi: arte orafa e miniatura sotto cristallo nel '200 e '300 veneziano, in L.CASELLI- E. MERKEL (curr.) , L'arte preziosa nel Veneto e a Venezia : un dialogo tra le arti figurative, Treviso [2007] pp. 85-101; M. SANTANICCHIA, La croce in cristallo di rocca del duomo di Foligno e la circolazione di opere veneziane in Umbria, in Arte medievale, 2010-2011, pp.121-142; J. TRIPPS, Ein Bücherschatz des Veneto in Leipzig : der Maestro di Giovanni da Gaibana und seine Bibeln aus dem Thomasstift, in T. FUCHS ; CH. MACKERT (Hrsg. 3 x Thomas, die Bibliotheken des Thomasklosters, der Thomaskirche und der Thomasschule im Laufe der Jahrhunderte; Leipzig,- 2012, pp.37-51; S. SPIANDORE, Miniature veneziane sotto cristallo, l'altare portatile di Firenze e la croce di Foligno, in Rivista di storia della miniatura, 16, 2012, pp. 35-45; EAD., Miniature sotto cristallo e smalti traslucidi in un misconosciuto altare portatile di Malta, in Arte Veneta,71, 2014 [2015], pp. 224-237. Mi sono inoltre potuta avvalere del lavoro di tesi della mia allieva ALESSANDRA TESSARI, Opere dei “cristalleri “veneziani con miniature. Analisi e sviluppo storico di una produzione, Università Ca' Foscari, A.A. $2006-2007$. ${ }^{2}$ Capitolare De cristallariis edito da G. MONTICOLO, I Capitolari delle arti veneziane sottoposte alla Giustizia e poi alla Giustizia Vecchia,III, Roma, 1914, pp. 123-152.

${ }_{3}^{3}$ G. MONTICOLO, I Capitolari delle arti veneziane sottoposte alla Giustizia e poi alla Giustizia Vecchia,I, Roma, 1896, pp. 115-132. Il Capitolare degli Oresi risale al 1233: nel capitolo VI si cita il cristallo come materiale da non utilizzare per falsificare gemme e pietre preziose; divieto ribadito in una giunta del 1262. ${ }_{4}^{4}$ Per il Tesoro di San Marco i principali testi di riferimento sono: R. GALLO, Il tesoro di S. Marco e la sua storia, Venezia-Roma, 1967; H. HAHNLOSER (a cura di ), Il Tesoro di San Marco. Il tesoro e il museo, Firenze 1971; Venezia e Bisanzio (catalogo della mostra, Venezia 1974) Milano, 1974, pp. 17-88; Il Tesoro di San Marco,(catalogo della mostra, Venezia, 1986) Milano 1986; H. FILLITZ, G. MORELLO (a cura di ), Omaggio a San Marco. Tesori dall'Europa, Milano 1994. ${ }_{5}$ È il caso delle anfore di cristallo islamiche: HAHNLOSER, Tesoro op.cit. 1971 (nota 4) cat. nn. 123, 125 ; Tesoro San Marco op. cit. 1986 (nota 4) cat. n. 31-32). 


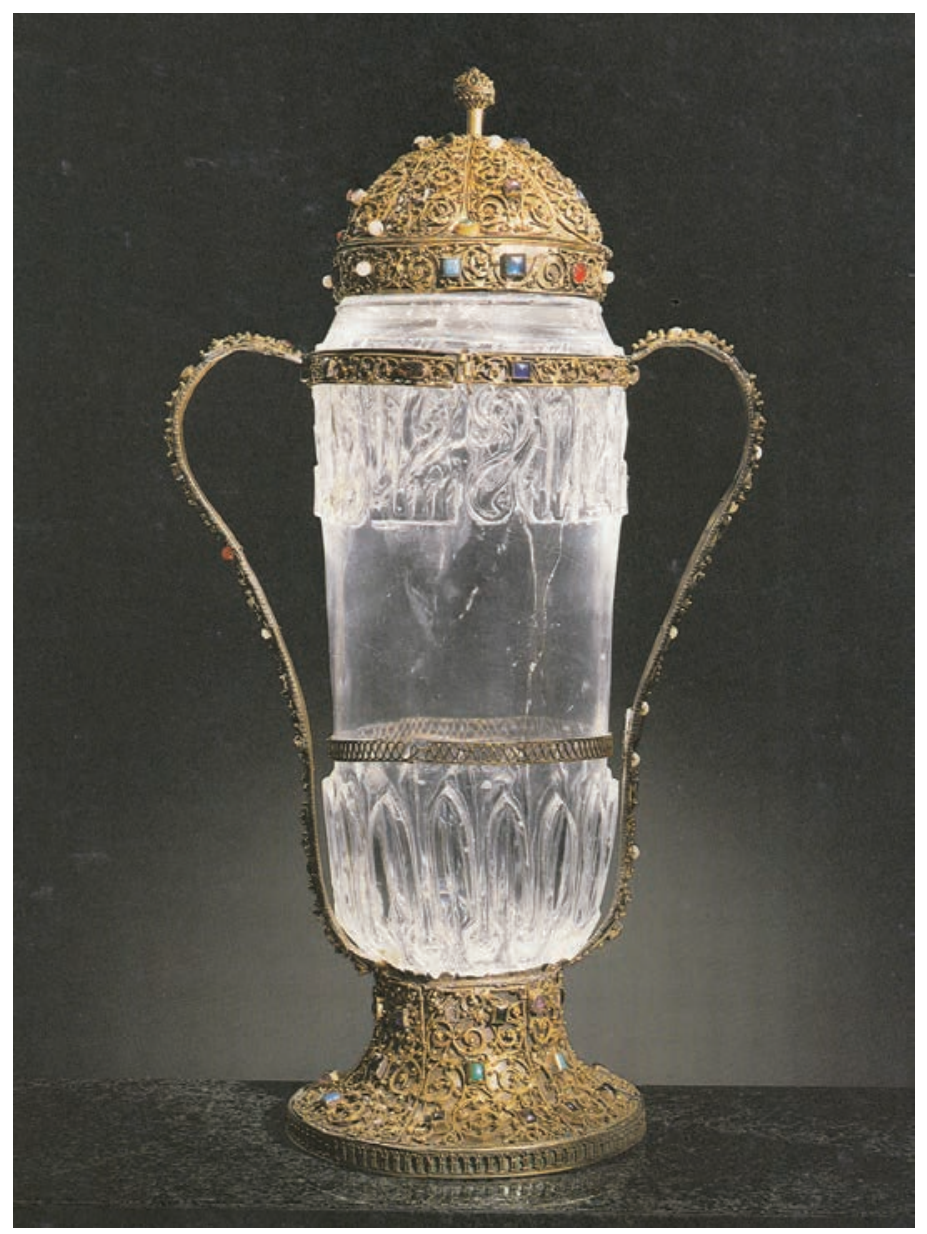

Fig.1. Venezia, Tesoro di San Marco, Vaso islamico in cristallo, montatura veneziana XIII secolo (foto da Tesoro di San Marco 1986 p.273).

una corona votiva bizantina in smalti. ${ }^{6}$ Nell'elaborazione di questa tecnica ebbe un ruolo fondamentale il confronto con la contemporanea produzione mosana e francese. ${ }^{7}$ Accanto a riusi e ricontestualizzazioni troviamo - sempre nel Tesoro marciano - i prodotti della nuova arte veneziana che assembla blocchi di cristallo molati e incisi per realizzare candelieri con basi e padelle in argento ${ }^{8}$. (Fig. 2) Questi saranno un prodotto di grande successo: una coppia ordinata da Carlo II d'Angiò è a San Nicola di Bari dal 1296, ${ }^{9}$ altri furono ordinati e acquistati o donati per altre chiese importanti, tra le altre

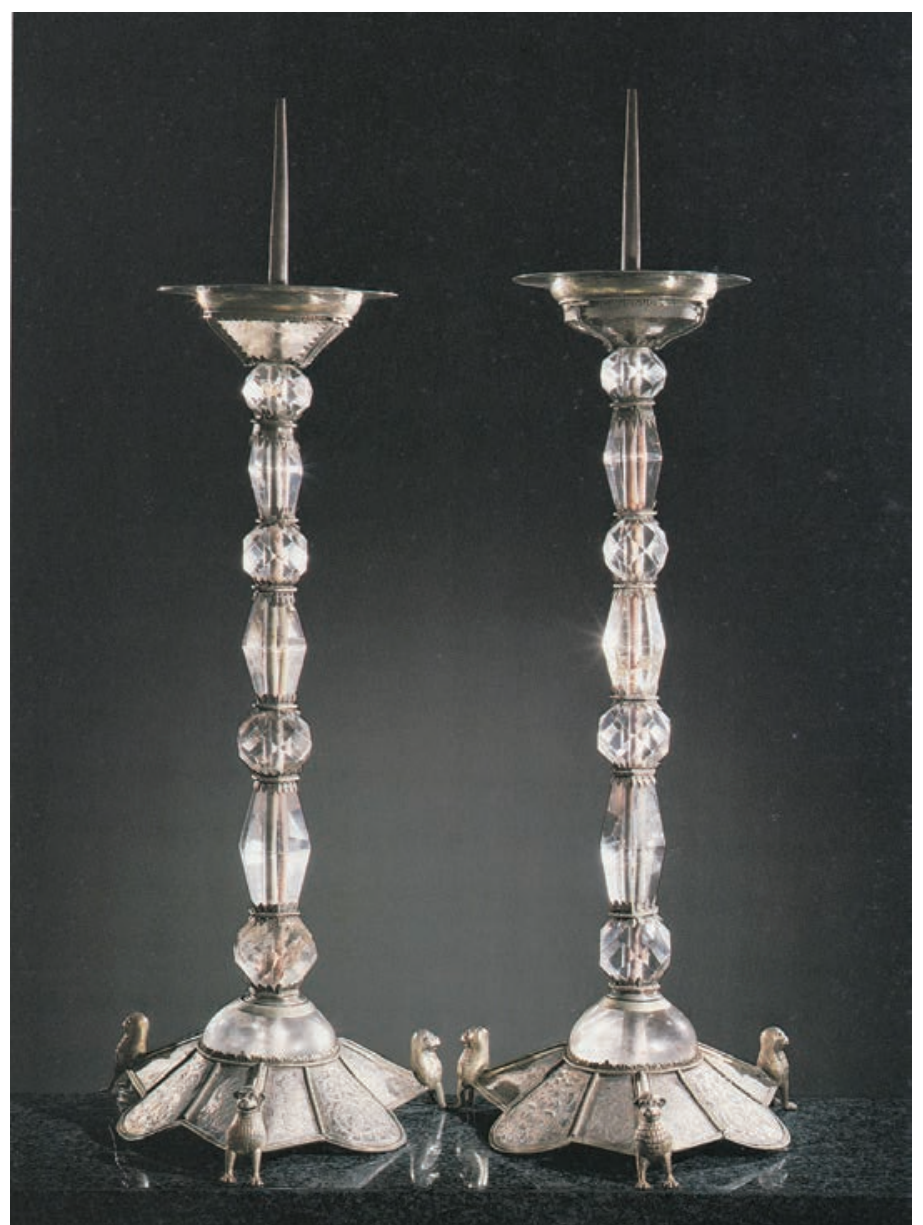

Fig. 2. Venezia, Tesoro di San Marco, Coppia di candelabri in cristallo di rocca (foto da Tesoro di San Marco 1986 p.283).

San Francesco di Assisi. ${ }^{10}$ Questo particolare elemento di arredo liturgico diventa quindi un prodotto di eccellenza delle officine veneziane che annoveravano però una varietà di oggetti elencati nel capitolare dell'arte:

«botoni, manici, roidi de boticelis et da ogli, tabule de anconis et de crucibus et lapides ad legendum $»^{11}$

Tra le preoccupazioni principali del capitolare del 1284 troviamo quella per il controllo sulle modalità di acquisto delle partite di cristallo, sulla sua distribuzione e riscontria-

\footnotetext{
${ }^{6}$ A. GRABAR in HAHNLOSER Tesoro op.cit. 1971 (nota 4), n.92 ; Venezia e Bisanzio op cit. (nota 4), n.25; M. E. FRAZER- D. GABORIT CHOPIN scheda 8 in Tesoro San Marco op.cit. (nota 4) 1986 , pp. 125-131; R. POLACCO, Cristallo di rocca, in EAM, V, Roma, 1994, pp.487-491:487.

${ }^{7}$ HAHNLOSER, BRUGGER KOCH, op. cit.(nota 1), p. 26; L. DOLCINI, La fortuna del cristallo di rocca nel Medioevo. Guida alla consultazione della bibliografia, in T. HACKENS , G. MOUCHARTE (ed.) , Technology and analysis of ancient gemstones, Rixensart 1989, pp. 341-368: in partic. p. 354; L. CASELLI, L'ornamento op. cit. (nota 1), p. 8o; M. COLLARETA, Vademecum per le arti suntuarie, in G.CAPUTO, G. GENTILI (a cura di ), Torcello, alle origini di Venezia tra oriente e occidente, Venezia, Marsilio 2009, pp. 116- 131: p. 122.

${ }^{8}$ Per i candelabri di cristallo e argento del Tesoro di San Marco: HAHNLOSER, Il tesoro op. cit. (nota 4), pp. 149-151; D. ALCOUFFE, D.GABORIT CHOPIN, schede 38-39 in Tesoro op. cit. 1986 (nota 4) pp. 282-285; R. POLACCO, op.cit.(nota 6), p. 491; E. MERKEL, scheda 26. Orafo veneziano, Due candelieri in rame argentato, argento sbalzato, niellato e dorato, composti ciascuno da nove elementi di cristallo di rocca, in Restituzioni 2004, tesori d'arte restaurati, Vicenza 2004, pp. 148- 151. Sull'apparizione di questa produzione a Venezia nel XIII secolo: H. R. HAHNLOSER, Opere di tagliatori veneziani di cristallo di rocca e di pietre dure del medioevo in Toscana, in Civiltà delle arti minori in Toscana, atti del convegno Arezzo, 11-15 maggio 1971, Firenze 1973, pp. 155-159; S. BETTINI, Saggio introduttivo, in Venezia e Bisanzio (catalogo della mostra, Venezia 1974), Milano 1974, pp. 17-88 (pp . 83-84 per cristalleri); ID., Venezia, nascita di una città, Milano 1978, pp. 187-188; HAHNLOSER- BRUGGER KOCH, Corpus op.cit. (nota 1), pp.26-27; D. ALCOUFFE, La glittica antica, bizantina e occidentale, in Il Tesoro di san Marco op.cit.(nota4) 1986, pp. 81-84.

9 I candelabri donati da Carlo d'Angiò alla chiesa di San Nicola di Bari sono lì attestati dal 1296 : P. TOESCA, Il Medioevo, II, Torino 1965, p. 1150-1151; HAHNLOSER, Tesoro op. cit. (nota 4), p.133; HAHNLOSER, BRUGGER KOCH op.cit. (nota 1) n.518, pp. 240-241; A. LAIOU, Venice as a Centre of Trade and of Artistic Production in the Thirteenth Century, in H. BELTING (a cura di), Il Medio Oriente e l'Occidente nell'arte del 13. secolo, Bologna 1982, pp. 1126 (partic. p.19); POLACCO op.cit. (nota 6), p. 491; V.PUGLIESE, scheda n. 20 in N. MILELLA, V.PUGLIESE (a cura di), Cittadella nicolaiana, un progetto verso il 2000, Bari 1995, pp. 185-190; M.L. FOBELLI, Circolazione artistica nel golfo di Venezia: la croce in cristallo di rocca del Museo Capitolare di Atri, in D.BENATI, A. TOMEI (a cura di), L’Abruzzo in età angioina: arte di frontiera tra Medioevo e Rinascimento , Cinisello Balsamo [2005], pp. 167- 179: 169,171. ${ }^{10}$ HAHNLOSER, BRUGGER KOCH, op.cit. (nota 1),n.517, p. 240; FOBELLI, op. cit. (nota 9), p. 169; SANTANICCHIA op. cit. (nota 1), pp.121-122.

${ }^{\mathrm{n}}$ MONTICOLO, Capitolare op.cit. (nota 2) : capitoli XXXX, XXXXI, p.133.
} 


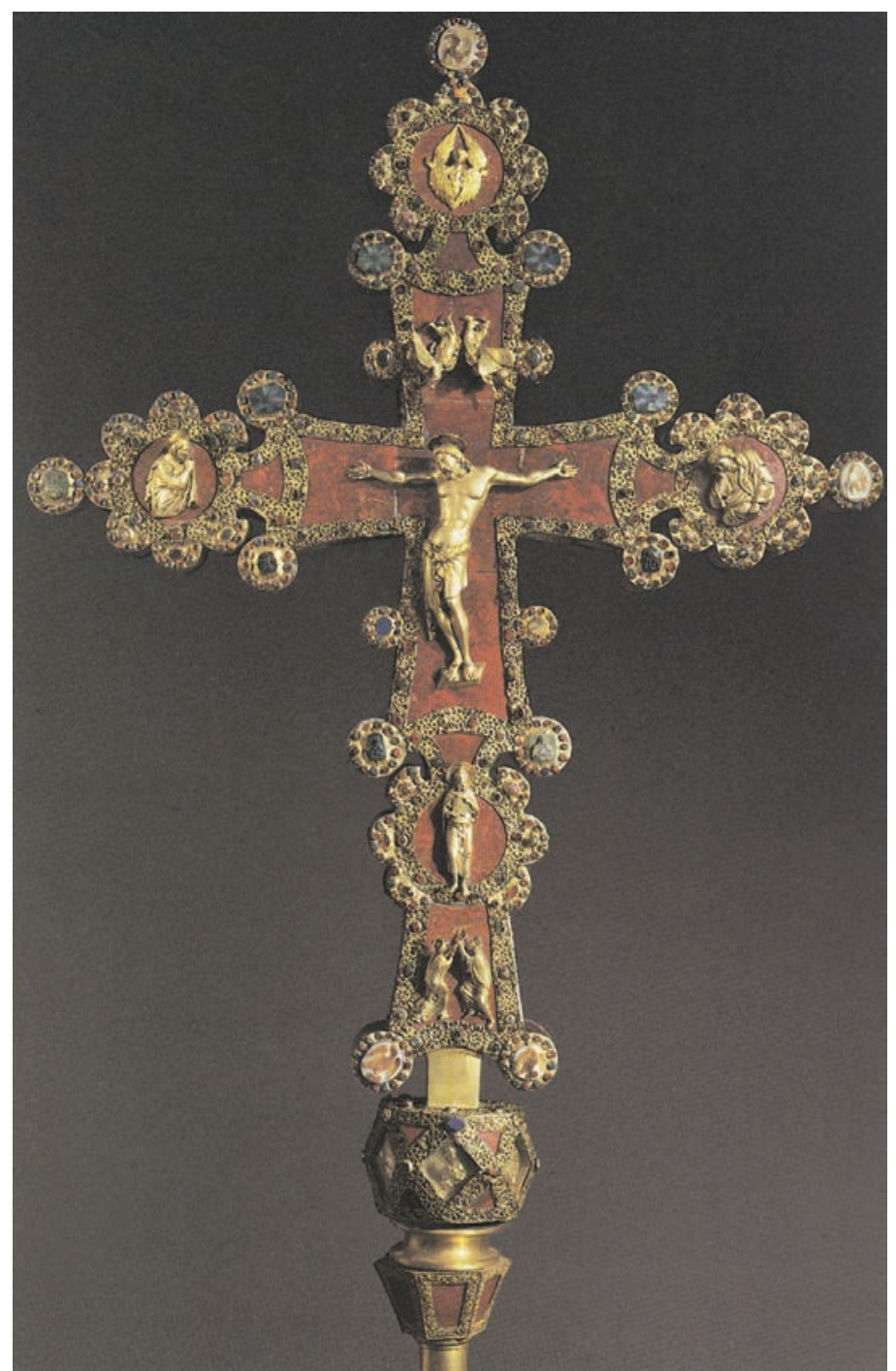

Fig. 3. Milano, Tesoro del Duomo, Croce di Chiaravalle (foto da CASELLI, 2002, p. 120).

mo l'attenzione fortissima alle contraffazioni, vietatissime sia per il mercato interno che per l'esportazione. ${ }^{12}$ Da queste norme ricaviamo immediatamente come i prodotti di questa tecnica siano destinati in misura rilevante al mercato ester- no. Gli acquisti di cristallo avvengono in consistenti quantità e sono sottoposti a verifiche per controllare evidentemente la quantità utilizzata in rapporto all'acquisto e evitare che vengano utilizzati vetri bianchi adulterando e frodando. Accanto al cristallo troviamo citati gli altri materiali utilizzati e tra questi principalmente il diaspro. ${ }^{33}$ Si tratta di un cristallo rosso venato che troviamo accostato al cristallo trasparente in tavole e in croci, ma che in qualche caso assume ruolo principale nella strutturazione dell'opera, come è il caso della croce di Chiaravalle. ${ }^{14}$ (Fig.3)

Nel capitolare nulla si dice dei metalli (argento, oro, ma anche rame) che costituiscono il legante essenziale, il connettore tra le parti tagliate, molate, levigate e assemblate secondo forme innovative. Neppure si fa cenno nel capitolare ad un'altra componente determinante in molti prodotti usciti dalle mani dei cristalleri : l'inserimento di miniature poste sotto le lastrine di cristallo che riproducono l'effetto ricercato volutamente - degli smalti. ${ }^{15}$ E' questa una tecnica attestata dalla cd croce di Desiderio a Brescia, ${ }^{16}$ nelle officine imperiali carolingie e dal trattato di Teofilo, che riemerge repentinamente a Venezia nel XIII secolo come componente qualificante della produzione dei cristalleri che si dovevano avvalere di maestri miniatori operanti a Venezia. ${ }^{17}$ Questo aspetto particolare è stato indagato a fondo, evidenziando una produzione ad hoc di immagini iconiche e narrative da parte di miniatori che culturalmente e formalmente si agganciano a vertici della produzione miniatoria veneta: dalle miniature dell'Epistolario del Gaibana di Padova (1259) fino ai messali prodotti nel XIV secolo per la basilica Marciana. ${ }^{18}$ Si tratta di diversi gruppi di miniatori che traducono in scala ridotta interi cicli e schemi iconografici elaborati in diversa misura, come i racconti musivi marciani. ${ }^{19}$ Le piccole immagini, impreziosite di perlinature e poste sotto le lastre cristalline, accostate e alternate a lastre di diaspro, incorniciate da fasce filigranate o sbalzate, incise, bulinate, qualificano gli oggetti informando sul contenuto prezioso, veicolando devozione e preghiere.

Si tratta infatti - nella maggior parte dei casi - di croci, reliquiari e tavole devozionali, includenti reliquie. Le miniature quindi illustrano le storie di Cristo, immagini mariane, figure di santi connesse ai luoghi di destinazione (San Fran-

\footnotetext{
${ }_{12}$ Ibidem.

${ }^{13}$ Ibidem, capit. XXXXV, p. 135 (giunta del 24 ottobre 1307): «quilibet de arte qui emerit iaspum ultra unum centenarium teneatur dare partem cuilibet magistro de arte partem volenti.» Il controllo sulle quantità era finalizzato anche a evitare contraffazioni.

${ }^{14}$ C. BERTELLI, La croce di Chiaravalle, in Il re dei confessori, dalla croce dei Cloisters alle croci italiane, Milano 1984, Pp.121- 131; L. CASELLI, La croce di Chiaravalle milanese e le croci veneziane in cristallo di rocca, Padova 2002; L. CASELLI, L'oreficeria veneziana nel Duecento e osservazioni intorno ad un oggetto esemplare, in L. CASELLI- R. POLACCO (a cura di),L'arte preziosa nel Veneto e a Venezia: valorizzazione culturale e nuovi progetti, Padova 2003 , pp. 49-66; M. DE GIORGI, scheda 79 Croce di Chiaravalle, in CAPUTO, GENTILI op. cit. (nota 7), p. 178; M. COLLARETA, Vademecum op. cit. (nota 7): 122, 130. ${ }^{15}$ S. BETTINI, Saggio, op. cit. (nota 8), pp. 17-88 (pp. 83-84 per cristalleri); ID., Venezia, op. cit. (nota 8),, pp. 187-188 per cristalleri; ID., Le miniature dell'Epistolario di Giovanni da Gaibana nella storia della pittura veneziana del Duecento, in C. BELLINATI- S.BETTINI, L'Epistolario miniato di Giovanni Da Gaibana, Vicenza 1968, pp. 71-120; A. NEFF, Miniatori op.cit. (nota 1); R. DEGEN, op.cit. (nota 1); W. DORIGO Venezia romanica : la formazione della città medioevale fino all'età gotica, Venezia, 2003, p. 558; SPIANDORE, op. cit. (nota 1).

${ }^{16}$ C. BERTELLI, 502. Croce di Desiderio, in C. BERTELLI- G.P. BROGIOLO (a cura di), Il Futuro dei Longobardi. L'Italia e la costruzione dell'Europa di Carlo Magno, Milano 200o, pp.526-527; G. SENA CHIESA, La "Croce di Desiderio", in R. STRADIOTTI (a cura di), San Salvatore- Santa Giulia a Brescia: il monastero nella storia, Milano, 2001, pp. 355- 369; C. STELLA, scheda VIII.1 Croce di Desiderio, in C.BERTELLI, C. STELLA, M'illumino d'immenso: Brescia le Sante Croci, Milano 2001, p.122.

${ }^{17}$ L. DOLCINI, op. cit. (nota 7), pp. 353-356.

${ }_{18}^{18}$ BETTINI, Le miniature op.cit. (nota 15); G. MARIANI CANOVA, La miniatura, in F. FLORES D’ARCAIS (a cura di), La pittura nel Veneto. Le origini, Milano 2004, pp. 223-244: 232-234; EAD. Scheda 4 in G. BALDASSIN MOLLI, G.MARIANI CANOVA, F. TONIOLO (a cura di), Parole dipinte. La miniatura a Padova dal Medioevo al Settecento, Modena 1999, pp.47-51; SPIANDORE, op. cit. (nota 1), p. 35; F. L. BOSSETTO, Per il Maestro del Gaibana e il suo atelier: un gruppo di Bibbie, in Rivista di storia della miniatura, 2009, 13, pp. 5l-61; TRIPPS, op. cit. (nota 1).

${ }_{19}$ V. LAZAREV, Saggi sulla pittura veneziana dei secc. XII - XIV. La maniera greca e il problema della scuola cretese, in Arte Veneta, 1965, pp. 17-31; DORIGO, op. cit. (nota 15) p. 558.
} 


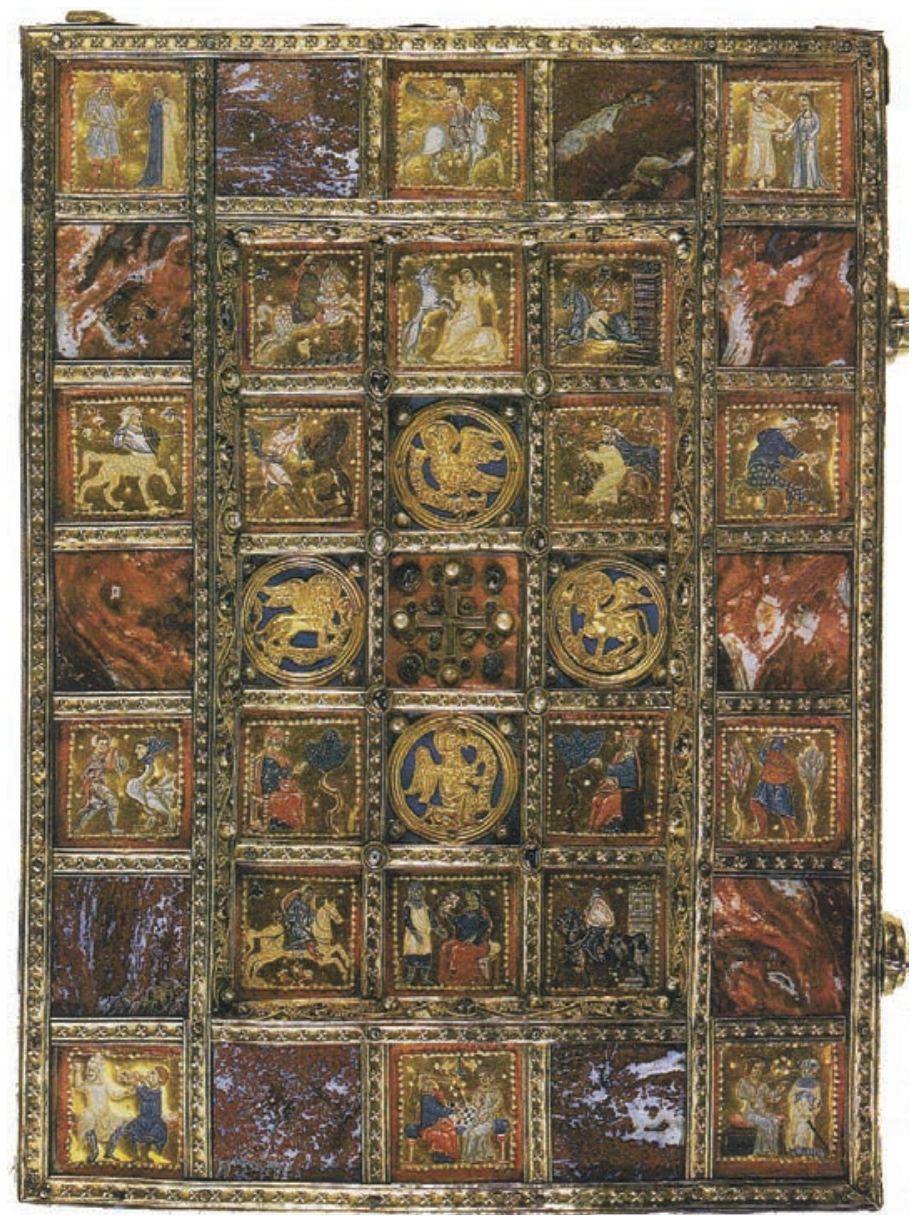

Fig. 4. Berlino, Kunstgewerbemuseum, Plenario di Ottone, inv. W32. cesco per chiese francescane) e al contenuto (Sant'Elena per le reliquie della croce).

Ma accanto a queste miniature di soggetto sacro possono trovarsi soggetti profani: è il caso del Plenario di Ottone der Milde, dal Tesoro dei Guelfi conservato a Berlino ${ }^{20}$ (Fig.4) e della scacchiera di Aschaffenburg. ${ }^{21}$ (Figg.5-6) Queste tavole presentano simile organizzazione con l'alternanza di caselle in diaspro rosso e figure sotto cristallo, queste ultime realizzate però con tecniche diverse (stucco per la scacchiera di Aschaffenburg e miniature per il Plenario di Berlino ${ }^{22}$ ) con soggetti profani, mostruosi e cavallereschi. La scacchiera è un oggetto non raro nella produzione dei cristalleri, che nel caso di Aschaffenburg ha acquisito un ruolo di reliquia per contatto, essendo considerata la scacchiera di San Ruperto. ${ }^{23}$ Già nel 1263 nel testamento di Pietro Vilio, un veneziano spintosi fino a Tabriz in Persia, ${ }^{24}$ si elencano tavole da gioco veneziane, con l'associazione di cristallo-diaspro - argento e perle destinate alla vendita su quelle vie orientali:

«[...]abeo in Turiso [Tabriz] apo mei: i’ abeo .j. tauleri dopio da gochare/a taule e le taule de quello tauleri; lo tauleri e le taule lavorate de christallo e di diaspado e d'argiento e di pietre e di perle; Ed ancho abeo . j. altro/tauleri dopio da gochare da l'uno lato a scachi e da l'altro a marelle, la quale tauleri este lavorato di christallo e di diaspado e di argento e di petre // e di perle; e li scachi e le marelle sono de christallo e di diaspado e questi .ij. tauleri soprascripti sono meçi di mio padre ser Vitale Veglone e meçi di ser Mafeo Miglano da Venesia». ${ }^{25}$

La tavola da gioco di Aschaffenburg- insieme ad un'altra proveniente dalla collezione dell'arciduca del Tirolo del castello di Ambras, conservata a Vienna ${ }^{26}$ - rappresenta la

\footnotetext{
${ }^{20}$ Berlino, Kunstgewerbemuseum, inv. n. W32; W. A. NEUMANN, Der Reliquienschatz des Hauses Braunschweig-Lüneburg, Hölder, Wien 1891, pp.249-256; O. von FALKE- R. SCHMIEDT- G. SWARZENSKI (hrsg.), Der Welfenschatz: der Reliquienschatz des Braunschwiger Domes aus dem Besitze des herzoglichen Hauses Braunschweig-Luneburg, Frankfurt a. M. 1930, pp. 82-83, 169-172, taf.76; Der Welfenschatz: : katalog der Austellung, Frankfurt a. M. 1930, pp. 15, 53-55; P. TOESCA, op. cit. (nota 1), pp. 15 -20; D. KÖTZSCHE, Der Welfenschatz im Berliner Kunstgewerbemuseum, Mann, 1973, cat. 51; HAHNLOSER, BRUGGER KOCH op.cit. (nota 1) n.25 pp. 86-87; D. KÖTZSCHE, Das Plenar Herzog Otto des Milden aus dem Welfen Schatz, Staatliche Museen Preussischer 1985, n.18; P.M. DE WINTER, The sacral Treasure of the Guelphs, in Cleveland Art Museum. The Bulletin of theCleveland Museum of Art, 72, 1985, pp. 3-16o: p. 124; J. M. FRITZ, Der Rückdeckel des Plenars Herzog Ottos des Milden von 1339 und verwandte Werke, in J. EHLERS , D. KÖTZSCHE (hrsg.), Der Welfenschatz und sein Umkreis Verfasserangabe, Mainz 1998, pp. 369- 385.

${ }^{21}$ HAHNLOSER, BRUGGER KOCH,op.cit. (nota 1), n. 50, pp. 94-95; F. KIRCHEWEGER, scheda 80 Tavoliere, in H. FILLITZ-G. MORELLO (a cura di ), Omaggio a San Marco. Tesori dall'Europa, Milano 1994, p. 97

${ }^{22}$ Nella sua tesi di laurea A. DALLA VILLA, Le miniature del Plenario del duca Otto. Arte profana veneta nel Tesoro dei Guelfi, Università Ca' Foscari, a.a. 2008 - 2009, ha condotto un serrato confronto con codici di letteratura provenzale riconducibili alla fine del XIII secolo e all'ambito veneto o dell'Italia settentrionale (in particolare il codice Saibante Hamilton 390 di Berlino) che concorrono a confermare l'attribuzione a Venezia della scacchiera.

${ }^{23}$ HAHNLOSER, BRUGGER KOCH,op.cit. (nota 1), p. 95.

${ }^{24}$ Il documento è stato pubblicato la prima volta nel 1883 ( B. CECCHETTI, Testamento di Pietro Vioni veneziano fatto a Tauris (Persia)MCCLXIV, X dicembre, in Archivio Veneto, XXVI, 1883, pp. 161-165), poi da E.BERTANZA- V.LAZZARINI, Il dialetto veneziano fino alla morte di Dante Alighieri 1321. Notizie e documenti, Venezia 1891, doc. n.41; e riedito da A. Stussi nel 1962 (A. STUSSI, Un testamento volgare scritto in Persia nel 1263, in Italia dialettale, 25, 1962, pp. 23- 37) che ne ha riveduto la data, la trascrizione e la valutazione dell'ambito linguistico di appartenenza, non tanto veneziano, ma pisano, dato che Vioni si avvalse di compagni di viaggio toscani come testimoni ed estensori. Si tratta di un testo ampiamente considerato negli studi sulla lingua italiana e sui commerci nel medioevo: M. F. TIEPOLO (a cura di), La Persia e la Repubblica di Venezia: mostra di documenti dell'Archivio di Stato e della Biblioteca Marciana di Venezia, Teheran 1973, pp.5-6; G. FOLENA, Culture e lingue nel Veneto medievale, Padova 1990, pp. 255-256; D.JACOBY, La Venezia d'oltremare nel secondo Duecento, in G. CRACCO- G.ORTALLI (a cura di), Storia di Venezia. II. L'età del Comune, Roma 1995, pp. 263-299: 274; D. JACOBY, Commercial exchange across the Mediterranean: Byzantium, the Crusader Levant, Egypt and Italy, Aldershot 2005, pp. 115, 119; Nel campo degli studi storico artistici : F. BRUNELLO, Arti e mestieri a Venezia nel Medioevo e nel Rinascimento, Vicenza 1981, p. 40; HAHNLOSER, BRUGGER KOCH, op.cit. (nota 1), pp. 27-28; ma soprattutto LAIOU, op. cit. ( nota 9), p.19. Il documento dimostra che la produzione di oggetti in cristallo, diaspro e perle è già una specifica dell'artigianato di lusso veneziano vent'anni prima del capitolare dei cristalleri: Pietro Vilio/Vilioni dispone infatti nel testamento circa le merci che ha con sé, in parte frutto di investimento di gruppi di veneziani, preoccupandosi di garantirne la realizzazione economica. Segnalo l'attuale collocazione del documento, mutata rispetto a quanto indicato da Cecchetti e Stussi: Venezia, Archivio di Stato, Procuratori di San Marco, Misti, perg. B.4.

${ }_{25}$ A.STUSSI, op.cit. (nota 24), p. 27. Oltre alle scacchiere, Vilio elenca altri oggetti (di altri veneziani a lui consegnati per commerciarli) in cristallo e diaspro: una sella da cavalcare, una fiala, due candelieri, una coppa con coperchio e altre due coppe. In coda al testamento indica al suo procuratore il valore dei pezzi: per ciascuna tavola da gioco 700 bisanti ( non meno di 6oo); la sella 6oo bisanti ; i candelieri 6oo bisanti; la fiala 9oo bisanti; la coppa con coperchio 150 bisanti; le altre due coppe per 225 bisanti (Ibidem, p. 29).

${ }^{26}$ Per la scacchiera di Aschaffenburg (Museen der Stadt, dalla collegiata dei SS. Pietro e Alessandro) ritenuta reliquia di San Ruperto, vedi nota 21 . Per la scacchiera di Vienna, che si differenzia dall'esemplare di A. perché accosta alle miniature sotto cristallo e al diaspro, inserti in agata, calcedonio e la lavorazione alla "certosina": NEUMANN, op.cit. (nota 20); HAHNLOSER, BRUGGER KOCH n. 51, p. 95; B. HÖLLANDER, cat. N. 76, in W. SEIPEL (hrsg.),
} 


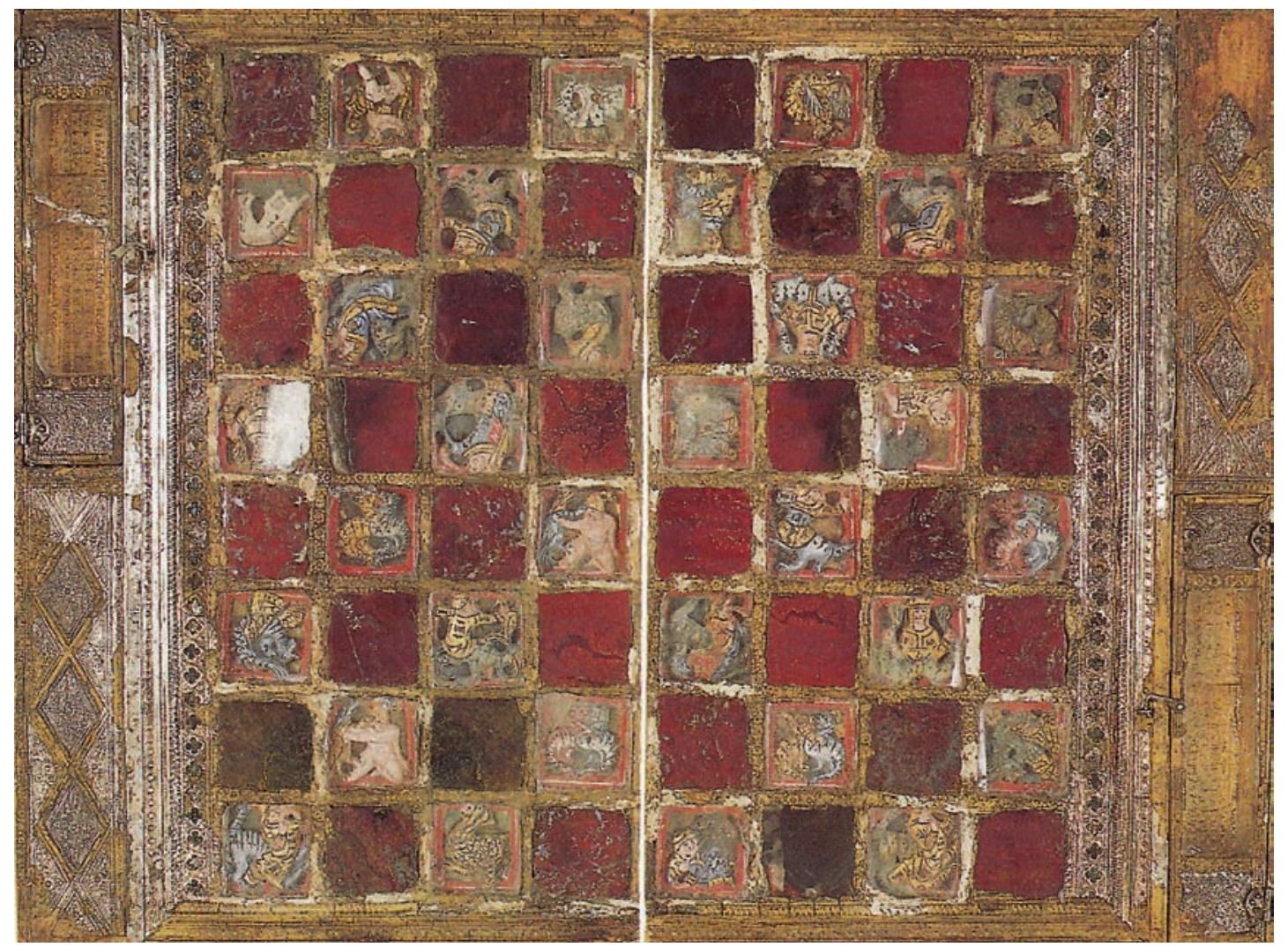

Fig. 5.
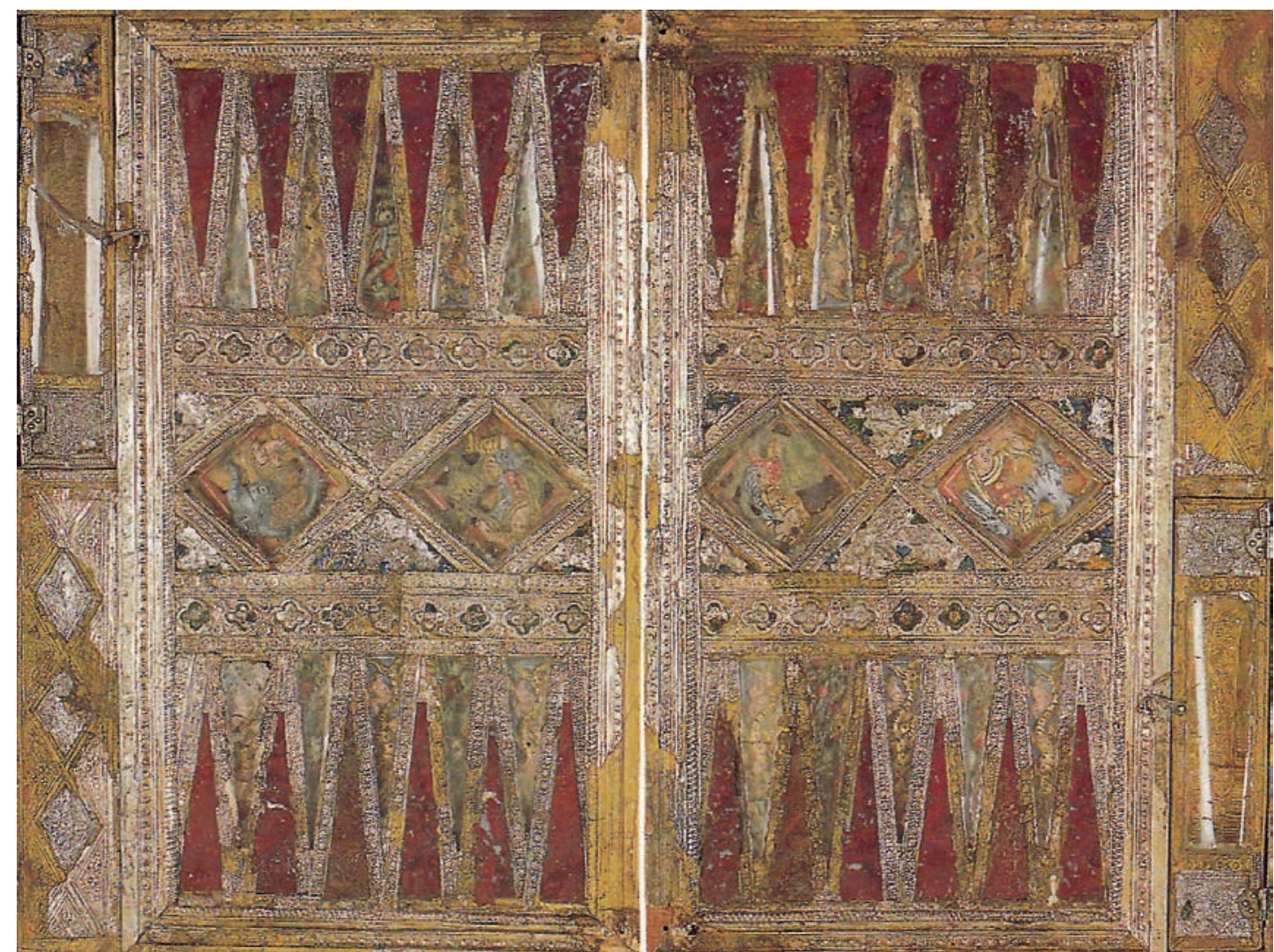

Figg. 5-6. Aschaffenburg, Museen der Stadt, scacchiera di San Ruperto, dalla collegiata dei SS. Pietro e Alessandro (foto da Omaggio a San Marco, p. 197).

sopravvivenza di questa produzione di lusso, destinata ad una clientela elevata. Anche la tavola del Plenario di Otto è organizzata come una scacchiera, con alternanza di caselle rosse e cristalline, con figure miniate riferibili al repertorio cortese ma non si tratta di una tavola da gioco, bensì di un piatto di legatura contenente una reliquia. Al centro appare

Spielwelten der Kunst. Kunstkammerspiele, Milano 1998, pp.152-153. Certamente le scacchiere presentano differenze che non consentono di raggrupparle e riferirle ad un unico ambito e momento produttivo. 


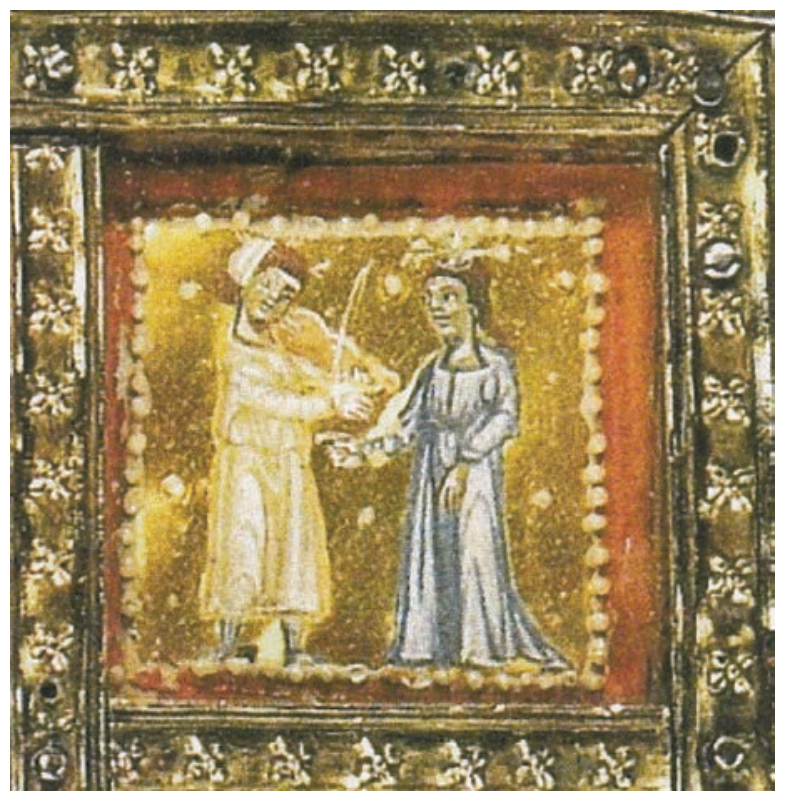

Fig. 7.

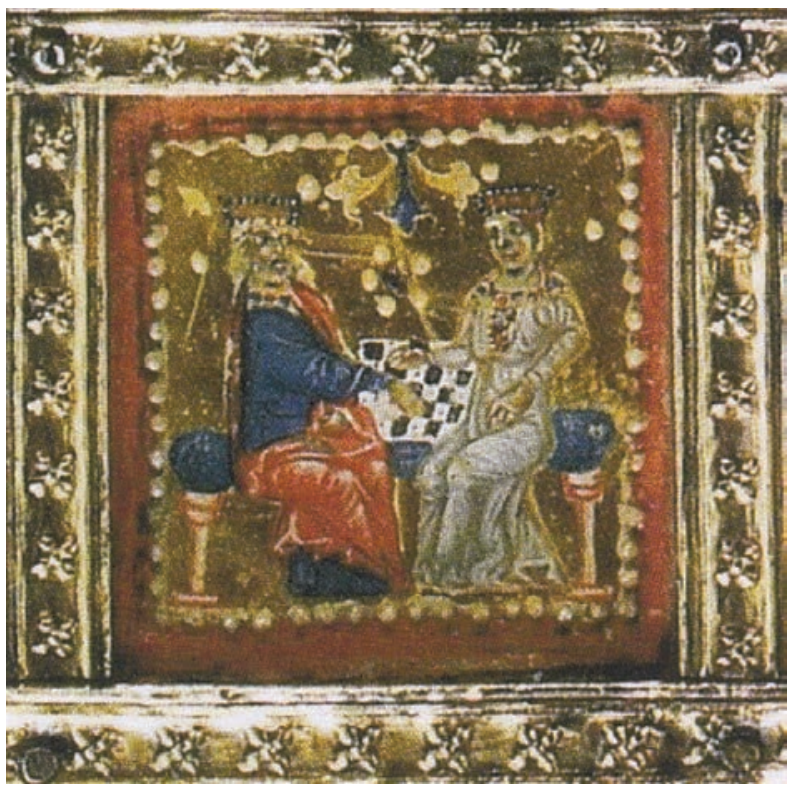

Figg. 7-8-9. Berlino, Kunstgewerbemuseum, Plenario di Ottone, inv. W32, particolari delle miniature sottocristallo

infatti una reliquia della croce circondata da figure a sbalzo e traforo con simboli degli evangelisti e la sequenza di diaspro e miniature sotto cristallo non rispetta una rigorosa alternanza ma segue logiche diverse, tese a rappresentare specularmente scene di vita cortese e avventure cavalleresche: musici e danzatori, lotte con animali fantastici, liberazioni di donzelle, omaggi cortesi; sapienti / reali in trono e una scena di gioco agli scacchi che vede protagonisti una donna e un

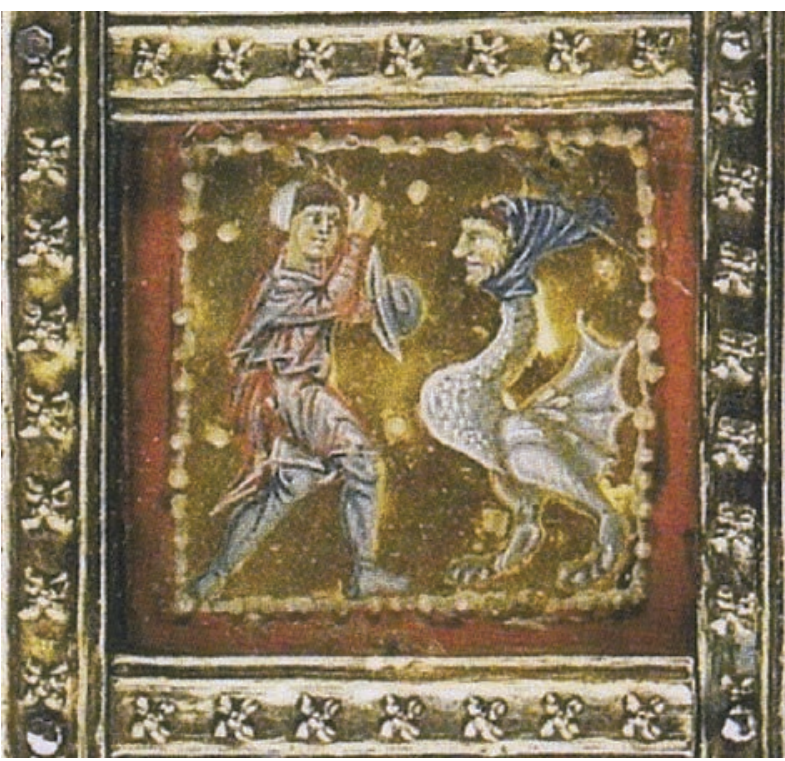

Fig. 8.

anziano barbuto. (Figg.7-8-9) La struttura a incasso centrale della tavola - con un motivo a tralcio nella modanatura interna - deriva dalla struttura di legature iconiche e reliquiari in tavole, ed è coerente con le fasce che incorniciano $\mathrm{i}$ riquadri in pietra e cristalli. Al momento della realizzazione del Plenario (1339) si trasformò una scacchiera in piatto di legatura con funzione anche di reliquiario; il numero delle caselle (dieci di diaspro e venti figurate) risulta inferiore a quello di una tavola da scacchi e quindi si può ipotizzare il sacrificio di alcune di esse e la riorganizzazione di quelle figurate al momento dell'inserimento della reliquia della croce e dei simboli degli evangelisti in nuove cornici di raccordo. ${ }^{27}$

Le scacchiere di Aschaffenburg, Vienna e la tavola utilizzata per il Plenario sono collocabili tra fine XIII e inizio del XIV secolo e sono accomunate dalla provenienza dall'area geografica tirolese e/o a famiglie nobili vicine o collegate agli Asburgo. La scacchiera di Vienna (parte della collezione del castello di Ambras, dell'arciduca del Tirolo) sarebbe collegata secondo la tradizione al duca Ottone di Carinzia e Tirolo ${ }^{28}$ mentre il Plenario di Berlino è legato alla coppia di committenti rappresentata nell'altro piatto, realizzato nel 1339: accanto a Ottone der Milden appare la moglie Agnese di Brandeburgo, imparentata con gli Asburgo e con i principi di Carinzia e Tirolo per parte di madre (Anna, figlia di Alberto I di Asburgo e di Elisabetta, figlia di Mainardo II, conte di Gorizia e Tirolo) ${ }^{29}$ e quindi nipote di Federico il Bello d'Austria, signore di Treviso per un breve periodo dal $1318-19 .^{30}$ Proprio alla famiglia dei principi di Carinzia e Tirolo possiamo ricollegare l'acquisto di scacchiere preziose a Venezia tra fine XIII e inizio XIV: dalla documentazione amministrativa dei principi, figli di Mainardo II, risulta infatti l'acquisto negli anni compresi tra il 1298 e il 1303 di

\footnotetext{
${ }_{27}$ NEUMANN, op. cit. (nota 20); J. von SCHLOSSER, Raccolte d'arte e di meraviglie del tardo Rinascimento, Firenze 1974, p. 31.

${ }^{28}$ NEUMANN, op. cit.(nota 20) , p. 253; SCHLOSSER, op.cit.(nota 27), p. 31. Potrebbe trattarsi del figlio di Mainardo II, conte di Gorizia e del Tirolo e quindi zio del Duca d'Austria Federico il Bello. Vedi J. HOERMANN, La famiglia di Mainardo II, in Il sogno di un principe: Mainardo II, la nascita del Tirolo, Mostra storica del Tirolo, Tirolo- Innsbruck 1995, pp. 165-170; G. TABACCO, La politica italiana di Federico il Bello re dei romani, in Archivio storico italiano, CVIII, 1950, pp. 3-77.

${ }^{29}$ Per la discendenza di Mainardo II, conte del Tirolo e Carinzia, fratello del conte di Gorizia Alberto II si vedano : J. RIEDMANN, L'area trevigiana e i poteri alpini in D. RANDO, G.M. VARANINI (a cura di), Storia di Treviso, II. Il Medioevo, Venezia 1991, pp. 243-267; J. HOERMANN, op. cit. (nota 28). Vedi Albero genealogico allegato. Il collegamento alla discendenza mainardina delle scacchiere di Vienna, Aschaffenburg, Berlino (riutilizzata nel Plenario) e del dittico di Berna (vedi oltre) era stato segnalato da NEUMANN op. cit. (nota 20), p. 253.

${ }^{30}$ TABACCO op. cit. (nota 28).
} 

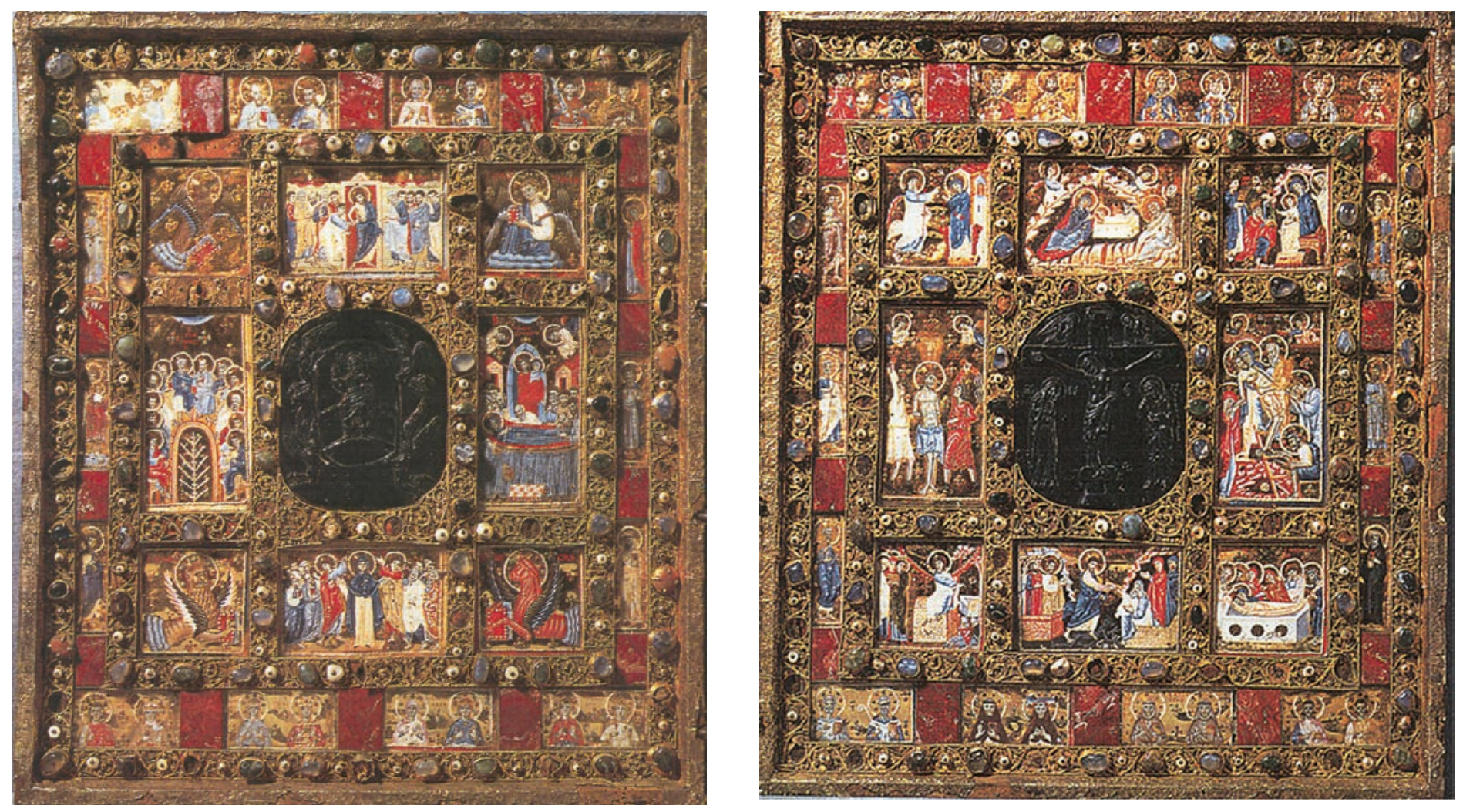

Fig.10-11 Berna, Museo Storico, Dittico di Andrea III d'Ungheria.

un numero impressionante di beni di lusso. Oltre a stoffe preziose, pellicce, perle, pietre e coralli tra il 1299 e il 1300 vengono acquistate quattro scacchiere. Gli emissari (Gottescalco e Niger da Trento, che facevano base a Mestre, la località testa di ponte del trevigiano sul fronte interno della laguna ) registrano le spese di imballo, trasporto e «rivatico in domo theutonica in Venetiis», egrazie a ciò abbiamo prova certa dell'acquisto a Venezia e - insieme - testimonianza del flusso dei materiali preziosi realizzati a Venezia. ${ }^{31}$
I cristalleri veneziani quindi collocavano i loro prodotti profani - destinati all'intrattenimento lecito del gioco di strategia considerato prerogativa dei nobili $3^{2}$ - sia sui mercati pericolosi e remoti d'oriente, ${ }^{33}$ sia sulla piazza veneziana, per una clientela in gran parte straniera. Di questa produzione non sopravvive nulla a Venezia, tranne forse un pezzo da gioco in cristallo conservato nel Tesoro di San Marco. ${ }^{34}$

E’ da notare che anche il Dittico di Berna35 (Figg.10-11) che apparteneva al re d'Ungheria Andrea III, figlio di una

31. RIEDMANN, Die Beziehungen der Grafen und Landesfuersten von Tirol zu Italien bis zum Jahre 1335, Wien 1977, pp. 270-274 per gli acquisti veneziani dei principi , in particolare p.270 per gli acquisti di scacchiere : nel 1299 una scacchiera, spezie, stoffe, perle, coralli, oggetti d'oro e d'argento, smeraldi, zaffiri, granati; un anno dopo risultano acquisti di gioielli, stoffe, pellicce, 20.000 coralli traforati, perle dorate, 300 zaffiri, 1.300 granati, 9 bussolotti in cristallo e tre scacchiere uguali. Per gli acquisti a Venezia da parte dei principi del Tirolo (che - insieme ad altri massicci acquisti a Firenze - li condussero al tracollo finanziario) vedi anche: J. RIEDMANN, La Marca e Venezia nella politica dei Conti di Gorizia e dei Conti del Tirolo (secoli XIII-XIV), in G. ORTALLI, M. KNAPTON (a cura di ), Istituzioni, società e potere nella Marca trevigiana e veronese (secoli XIII-XIV). Sulle tracce di G.B. Verci, Roma 1988, pp. 361-367 (in particol. p. 367); ID., L'area trevigiana op. cit. (nota 25), pp. 250-253. Fin dalla prima metà del XIII secolo i conti di Gorizia avevano rapporti economici con Venezia : R. HARTEL, Il commercio veneziano con il Friuli e con il retroterra austriaco intorno al 120o, in Atti Istituto Veneto Scienze Lettere e Arti, CLIV, 1995-1996, pp. 579-607, in particolare p. 594.

${ }^{32}$ R.A. MÜLLER, IL gioco degli scacchi come metafora della società tardomedievale, in Ludica, 1, 1995, pp. 114- 125; A. RIZZI, Ludus/ludere. Giocare in Italia alla fine del Medioevo, Roma 1995, pp. 63-66; L. GENOVESE, Dadi, scacchi e tric-trac: la ludicità nel Mezzogiorno normanno, in Ludica, 11, 2005, pp. 19 - 27. Nel 1312 Rizzardo da Camino, signore di Treviso viene assassinato mentre gioca a scacchi (G.M. VARANINI, Istituzioni e società tra Comune, Signoria e poteri regionali (1259-1339), in D.RANDO, G.M.VARANINI (a cura di), Storia di Treviso, II, Il medioevo, Venezia 1991, pp. 135- 211, in partic. p. 177 ).

In realtà il gioco era diffuso anche tra i popolani: G. ORTALLI, Et occasione ludi venerunt ad verba. Una partita a scacchi finita male nella Venezia duecentesca, in S. PERINI (a cura di), Tempi, uomini ed eventi di storia veneta. Studi in onore di Federico Seneca, Rovigo 2003, pp. 127 - 132.

Sulla rappresentazione del gioco degli scacchi con valenza moraleggiante si veda: G. TROVABENE, Gioco, virtù e vizio in un testo musivo medioevale, in Venezia arti, 15/16.2001/02(2005), pp. 33-42.

Con riferimento all'area nobiliare oltremontana si può richiamare anche il Codex Manasse, il magnifico codice con illustrazioni di ambito cortese e cavalleresco (1305-1340) dove sono rappresentate due scene di gioco: il margravio Ottone di Brandeburgo mentre gioca a scacchi con una dama e una partita a tric-trac tra due gentiluomini (Universitätbibliothek Heidelberg, Cod. Pal. Germ. 848, fol. 13 r e 262v , Bild-ID HeidIcon 12696 e 12780).

33 Vedi il testamento di Pietro Vilio, nota 24.

34 Il pezzo è riferito dubitativamente all'ambito islamico in : C. J. LAMM, Mittelalterliche Glaser und Steinschnittarbeiten aus dem Nahen Osten , Berlin 1929-30, p.206-207, n. 73.1; K. ERDMANN, cat. n.129, in HANHLOSER, op.cit. (nota 4), p. 118 ; F. GABRIELI, U. SCERRATO (a cura di), Gli arabi in Italia, cultura, contatti, tradizioni, Milano 1993, p.517 e fig. 586. Per questo pezzo POLACCO, op. cit. (nota 6), p. 488, ha proposto una datazione altomedievale sulla base del motivo a losanghe: piuttosto - a mio avviso - questo motivo è confrontabile con le losanghe della tavola da gioco di Aschaffenburg, ma anche con l'altarolo Pitti, per il quale vedi: L. MORETTI, scheda 8o in Venezia e Bisanzio, Milano 1974; HAHNLOSER, BRUGGER KOCH op. cit. (nota 1), n.10, p. 81; SPIANDORE op.cit. (nota 1), pp. 37-40, 44.

35 Berna, Museo Storico, TOESCA op. cit. (nota 1); BETTINI, Le miniature op. cit. (nota 15), pp. 115-116; HAHNLOSER, BRUGGER KOCH, op. cit. (nota 1), n.23, pp. 85-86; NEFF op.cit. (nota 1), p. 7; LAIOU op. cit. (nota 9), pp. 20, 22; DEGEN op. cit. (nota 1), pp. 449-465; FOBELLI, op. cit. (nota 9), pp.168-169; SPIANDORE op. cit. (nota 1) , pp. 35, 41- 42; TRIPPS, op. cit. (nota 1). 
MAINARDO I

conte del Tirolo -Carinzia

$(+1258)$

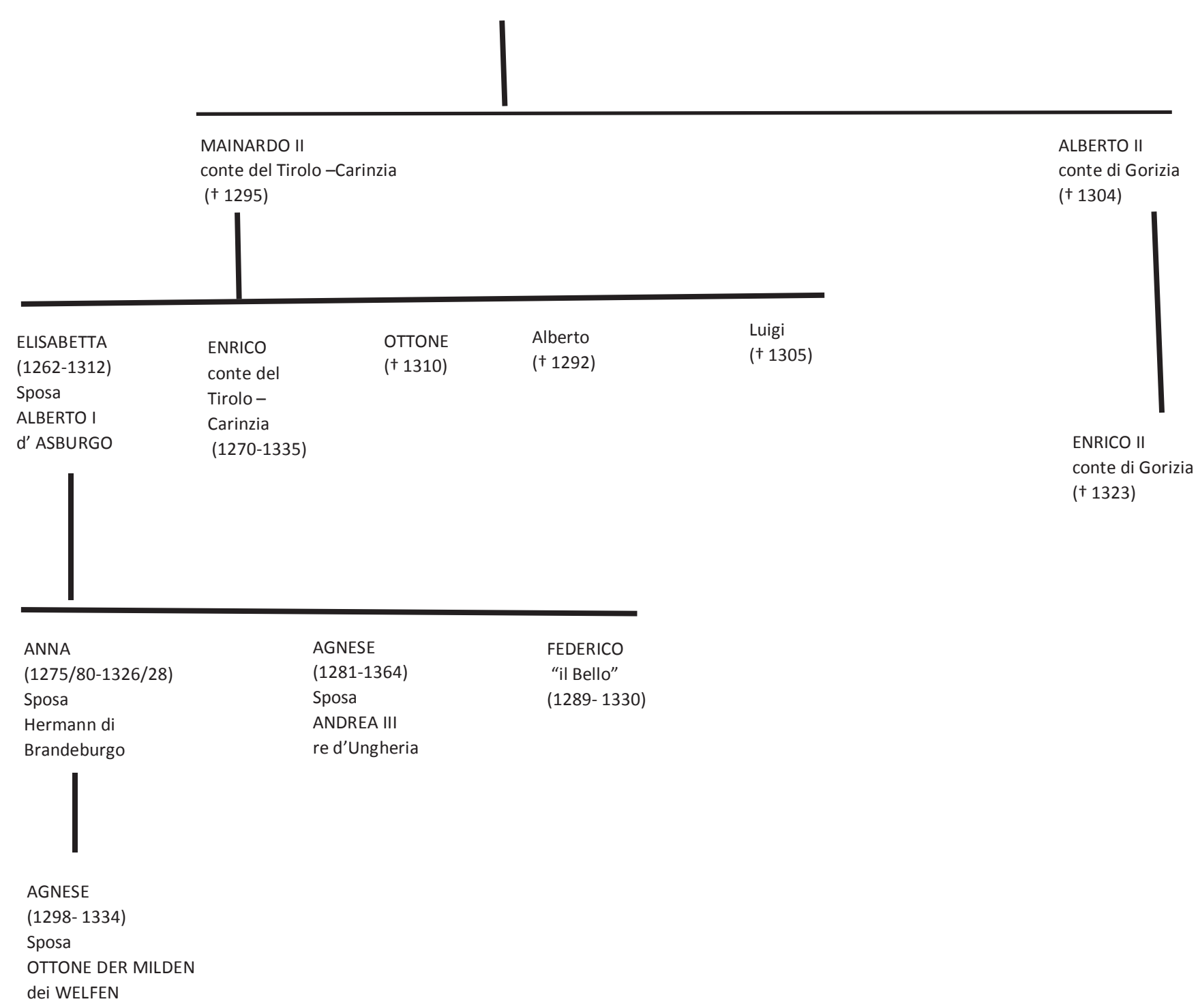

veneziana (Tomasina Morosini), si ricollega all'ambito degli Asburgo e dei discendenti di Mainardo II, poichè la regina d'Ungheria, sposata nel 1296, Agnese (la quale donerà il dittico al monastero di Konigsfelden) era figlia di Alberto I d'Asburgo e di Elisabetta di Carinzia e quindi sorella di Federico d'Austria e nipote dei principi mainardini. ${ }^{36} \mathrm{Il}$ dittico di Berna è un oggetto riferibile alla devozione privata dei reali d'Ungheria e declina l'associazione di miniature, cristalli e diaspri con l'incorniciatura di filigrana e gemme incastonate in una trama di rettangoli e quadrati. Le miniature sono organizzate gerarchicamente: nel registro esterno appaiono figure di santi interi e a mezzo busto, mentre il quadro interno, a sua volta diviso in otto riquadri, accoglie scene della vita di Cristo intorno a due diaspri scuri di grande dimensione che occupano il centro delle tavole e rappresentano la Crocifissione e l'Ascensione. Si tratta di un'opera riferita - soprattutto per lo stile delle miniature - agli anni novanta del XIII secolo ${ }^{37}$ che può rientrare nella tipologia delle tavole d'ancona ricordate nel Capitolare dei cristalleri. Si tratta di un genere produttivo di cui sopravvivono altri esempi importanti conservati a Monte Athos, riferibili alla committenza dei re di Serbia. ${ }^{8}$ Anche in questo caso incontriamo dei regnanti imparentati con veneziani: Anna Dandolo (nipote del doge Enrico Dandolo conquistatore di

$3^{6}$ Vedi ALBERO GENEALOGICO allegato.

37 TOESCA, op.cit.(nota 1); BETTINI, Le miniature op. cit. (nota 15), pp.115- 116; NEFF, op. cit. (nota 1), p. 7; MARIANI CANOVA , La miniatura op. cit. (nota 18), pp. 235- 237.

${ }^{38}$ Per i due dittici del Monte Athos (Biblioteca Hagiou Paulou e Biblioteca Chilandari) vedi: HAHNLOSER, BRUGGER KOCH, op. cit. (nota 1), nn.21-22, p. 85; P. HUBER, Image et message: miniatures byzantines de l'Ancien et du Nouveau Testament, Zürich 1975, pp. 115-199; LAIOU op. cit. (nota 9), pp.20, 22; K. LOVERDOU TSIGARIDA, Tresaures of Mont Athos, Thessaloniki 1997, pp. 323- 327; DEGEN, op. cit. (nota 1),pp. 428-448; CASELLI , L'ornamento op. cit. (nota 1) pp. 88-9o. 


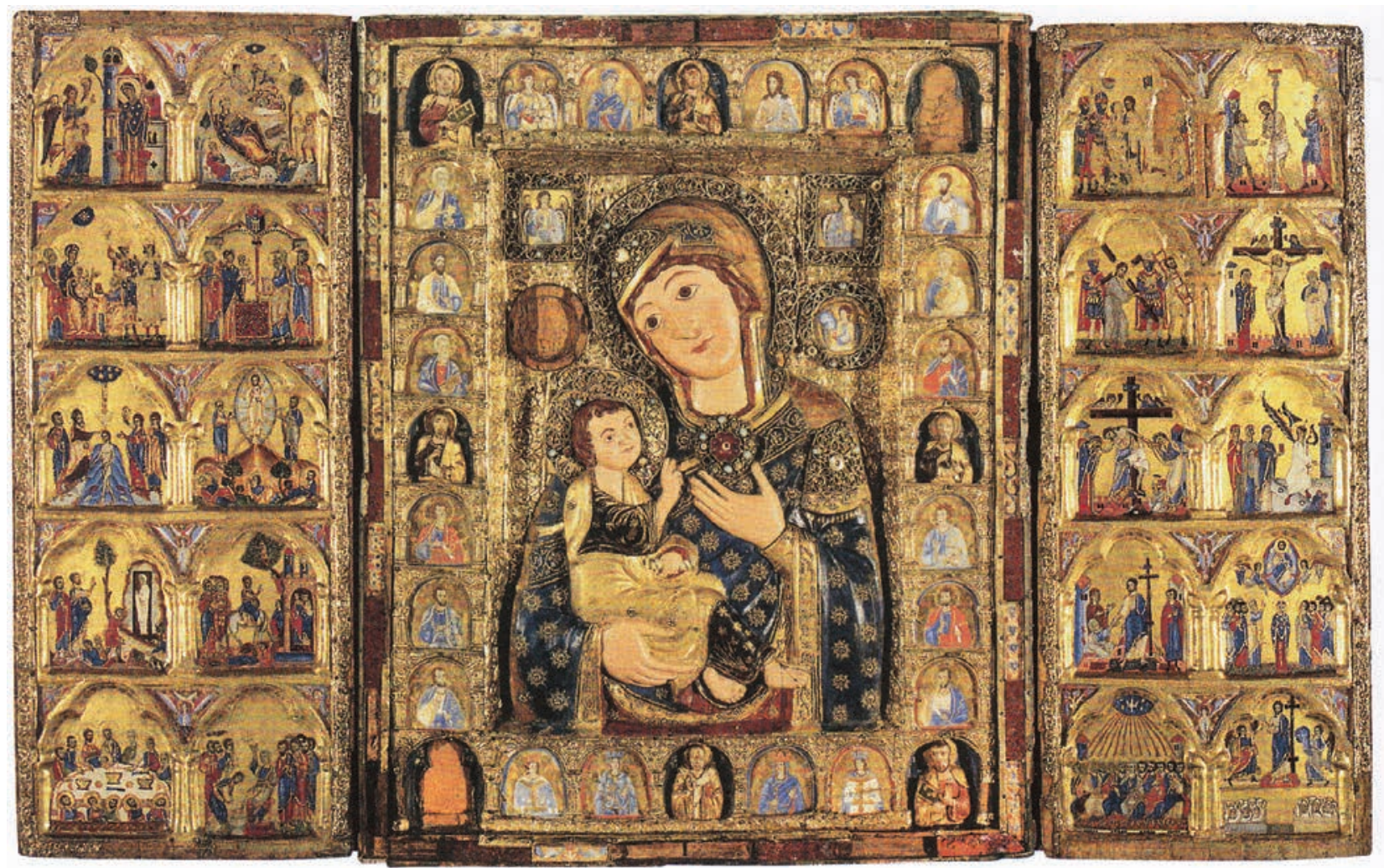

Fig. 12. Celano (L'Aquila), Museo nazionale d'Arte Sacra del Marsicano, Trittico di Alba Fucens (foto da CAPUTO-GENTILI, Torcello op.cit., p.160).

Costantinopoli) sposa nel 1217 il re di Serbia Stefano Uros ed è quindi madre del donatore di questi ogetti. ${ }^{39}$ Dittici di grande preziosità vengono quindi in mano a regnanti che molto probabilmente li avevano commissionati, e nel giro di pochi decenni vengono tesaurizzati e sacralizzati in ambiti ecclesiastici..$^{\circ}$

Tra le tavole d'ancona dobbiamo considerare anche il famosissimo trittico di Alba Fucens (Fig. 12) ${ }^{41}$ che rappresenta però una variante rispetto ai dittici di Berna e Monte Athos: poche placchette in diaspro alloggiano ai bordi della tavola centrale dove campeggia una Odighitria a rilievo in legno dipinto, circondata da filigrane e gemme e incorniciata da figure di santi in arcata, scolpiti e dipinti o miniati sotto cristallo. Le ante laterali sono tavole dipinte: troviamo qui una ulteriore declinazione di un'arte polimaterica che vedeva la collaborazione di artisti di diverse corporazioni per realizzare opere che rappresentavano una traduzione in materiali di minor costo e più abbordabili delle icone bizantine a sbalzo arrivate nel Tesoro marciano raffiguranti l'arcangelo Michele ${ }^{42}$. (Fig. 13)

Altri prodotti delle officine dei cristalleri di grande successo e diffusione sono le croci, realizzate fondamentalmente con lastrine rettangolari profilate alle estremità con polilobi, congiunte al centro in vari modi, con miniature sotto cristal-

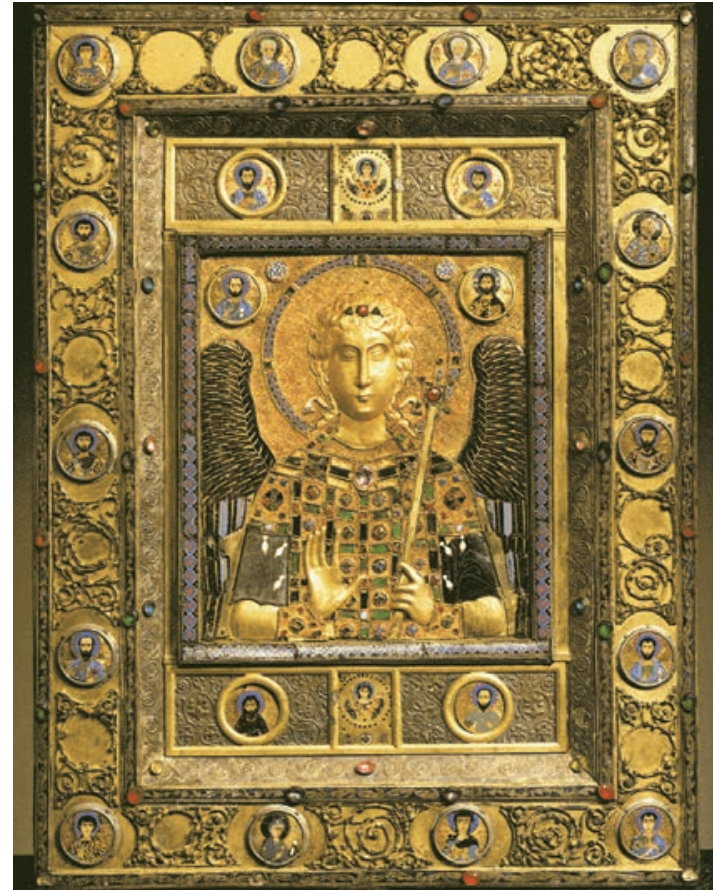

Fig. 13. Venezia, Tesoro di San Marco, Icona dell'Arcangelo Michele (foto da Tesoro di San Marco 1986 p. 72).

39 E. PASTORELLO (a cura di ), Andreae Danduli ducis Venetiarum Chronica per extensum descripta : aa. 46-128o d.C., Bologna, 1938 (RIS ,12/1), p. 287. ${ }^{40}$ Il dittico di Berna (vedi nota 35) viene infatti donato dalla regina d'Ungheria ritiratasi in convento a Konigsfelden; mentre i dittici di Monte Athos (vedi nota 38) sono doni dei re Stefano I e Stefano Uros II Milutin. CASELLI, L'ornamento op. cit. (nota 1), p. 89 ha segnalato anche la presenza di mercanti veneziani a Dubrovnik e quindi la possibilità di acquisti per quella via.

${ }^{4}$ Celano (L'Aquila), Museo naz.le Arte Sacra del Marsicano, già Roma, Palazzo Venezia, TOESCA, op. cit. (nota 1) p. 18-19; HAHNLOSER, BRUGGER KOCH, op. cit. (nota 1), n. 29, pp. 87-88; DEGEN op.cit. (nota 1) pp. 474-484; P. AMATO, Imago Mariae, tesori d'arte della civiltà cristiana, catalogo della mostra (Roma, 10 febbraio- 20 giugno 1988), Milano, Mondadori, 1988, pp. 98-99; F. KIRCHEWEGER, scheda 73 in H. FILLITZ, G. MORELLO op. cit. (nota 21), pp. 186- 187; M.G. BARBERINI in F. DE SANCTIS, A. SARAGOSA (a cura di), Testimonianze preziose, capolavori sacri svelano la loro storia, catalogo della mostra (Avezzano- Celano 2008), L’Aquila 2008. Il trittico faceva parte del Tesoro di Alba Fucens, donato dalla regina Giovanna I di Napoli († 1382), sposata in prime nozze con Andrea d'Ungheria e in quarte nozze con Ottone IV di Brunswick.

${ }^{42}$ A. GRABAR, in HAHNLOSER, Il Tesoro di San Marco op.cit. (nota 4) nn.16-17, pp. 23-27; B. DRAKE BOEHM, schede 12 e 19, in Tesoro op. cit. 1986 (nota 4), pp.149-155, 179-182; E. MERKEL, scheda 19. Orafo costantinopolitano, Icona dell'arcangelo Michele stante e ad ali spiegate, in Restituzioni 2004, tesori d'arte restaurati, Vicenza 2004, pp. 120-123; M. DE GIORGI, scheda 54 Icona dell'Arcangelo Michele, in CAPUTO, GENTILI (a cura di ) , op. cit. (nota 7 ) , p. 173. 


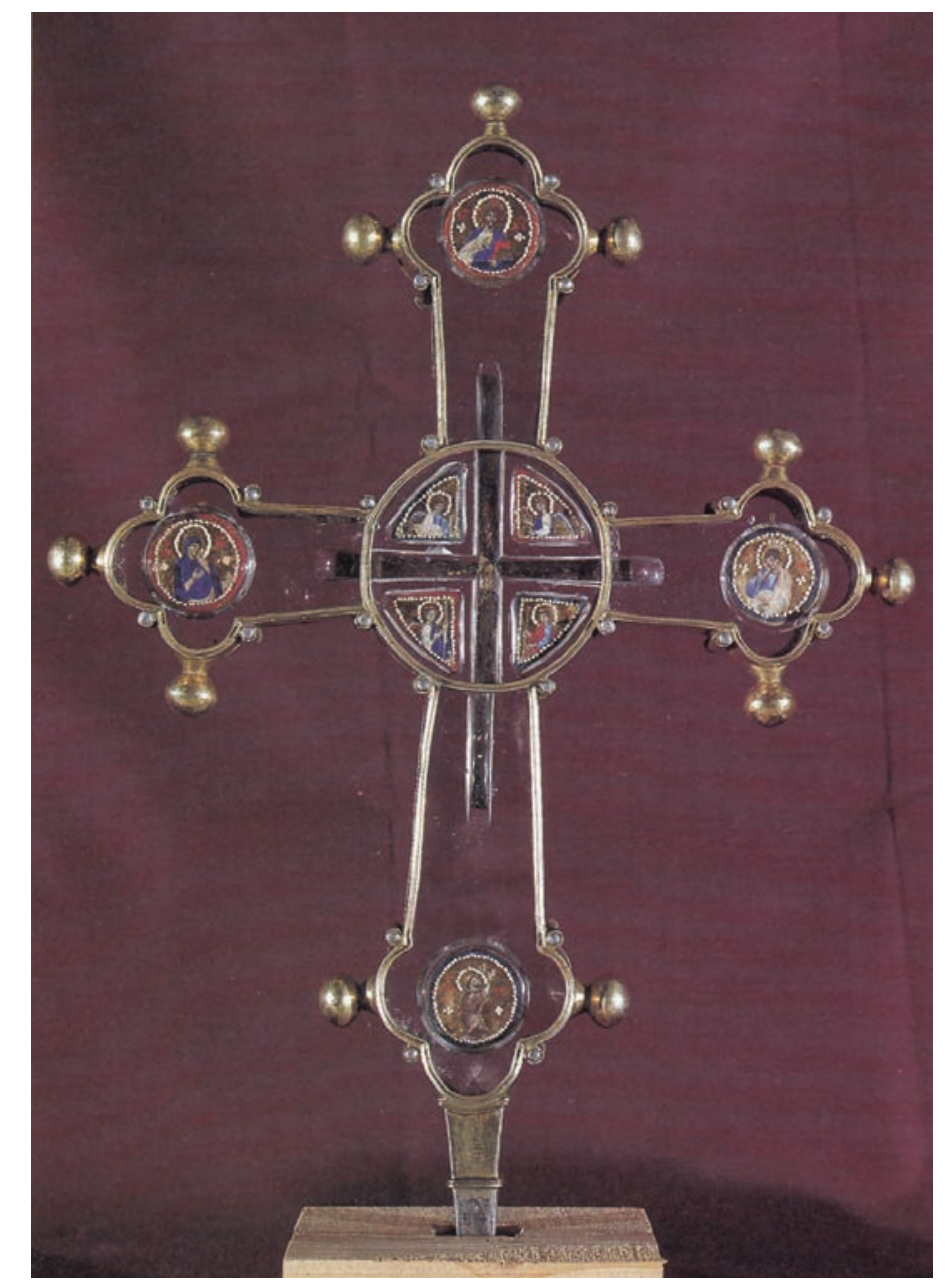

Fig. 14. Atri, Museo Capitolare, Croce reliquiario (foto da FILLITZ, MORELLO, Omaggio a San Marco, p. 199).

lo, e più avanti nel XIV secolo con smalti traslucidi (croce Assisi), la nuova tecnica che viene aggiunta alla gamma materica di questi oggetti complessi. ${ }^{43}$ Nella maggior parte dei casi le croci accolgono al centro una reliquia della croce che può essere a vista (Atri) (Fig. 14) o visibile sul verso (San Candido); ${ }^{44}$ in alcune la cella per reliquie è protetta da una lastrina di diaspro (Udine), 45 (Fig. 15) oppure celata in una teca metallica in forma di crocifissione (Foligno) ${ }^{46}$.

Si tratta di croci composte da lastre piatte, che definiscono direttamente i quattro bracci della croce, a volte arricchite da sfere costolonate alle estremità o all'incrocio, montate su un nodo che può essere un cristallo costolonato o sfaccettato oppure un poliedro di argento con diaspri e/o miniature o smalti, con variazioni che denotano una produzione non

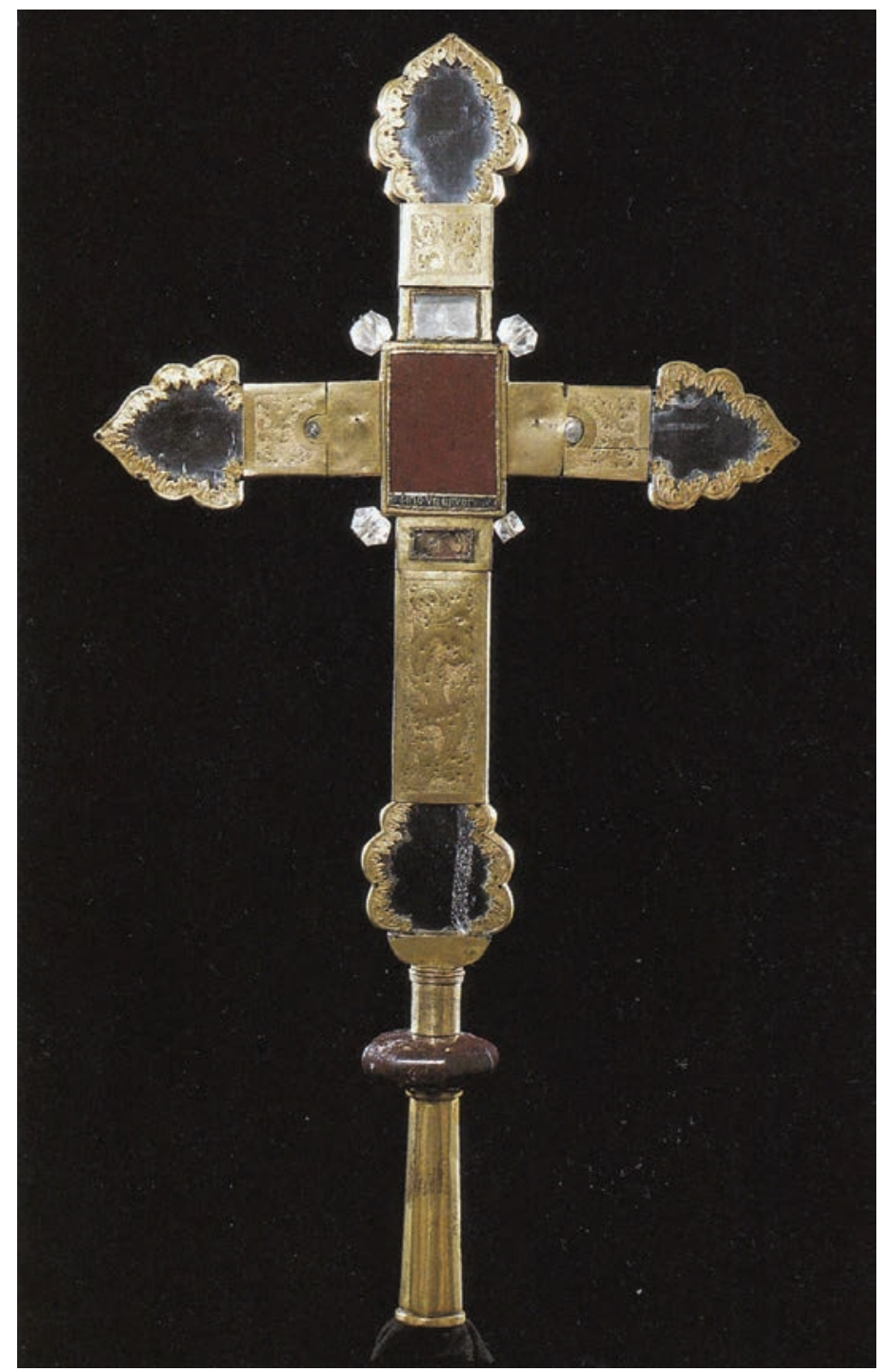

Fig. 15. Udine, Civici Musei, Croce reliquiario, verso (foto da FILLITZ, MORELLO, Omaggio a San Marco p.243).

seriale, basata su una gamma di varianti nei profili, nelle combinazioni e negli arricchimenti. Vi sono poi nel corpus dei cristalleri veneziani, croci più complesse, arricchite da terminazioni fortemente apicate e germinazioni, con miniature sotto cristallo che occupano tutta l'estensione dei bracci della croce (Hagiou Pavlou, monte Athos) ${ }^{47}$ oppure - con la stessa sagoma - la croce di Chiaravalle, interamente in diaspro su un lato e in argento a sbalzo sotto cristallo nell'altro, con cornici filigranate e germinazioni di gemme incastonate..$^{48} \mathrm{La}$ fronte in diaspro è decorata da micro-sculture di argento dorato che

43 Per la croce di Assisi vedi: HAHNLOSER, BRUGGER KOCH, op. cit. (nota 1), n. 66, p. 100; DEGEN op. cit. (nota 1), pp.187-200; SANTANICCHIA, op. cit. (nota 1), pp.128-132; COLLARETA, Il cristallo op.cit. (nota 1); CASELLI, L'ornamento op.cit. p. 96. Per l'introduzione dello smalto traslucido nell'oreficeria veneziana vedi : G. MARIANI CANOVA, Presenza dello smalto traslucido nel Veneto durante la prima metà del Trecento, in Annali della Scuola Normale Superiore di Pisa, Classe di Lettere e Filosofia, III Ser, 14, 1984, pp. 733-755; e da ultimo: S. SPIANDORE, Miniature sotto cristallo e smalti traslucidi in un misconosciuto altare portatile di Malta, in Arte Veneta,71, 2014 [2015], pp. 224-237.

${ }^{44}$ Per la croce di Atri: HAHNLOSER, BRUGGER KOCH, op. cit. (nota 1), n. 110, p. 116; DEGEN op. cit. (nota 1), pp. 326-333; F. KIRCHEWEGER, scheda 82, in FILLITZ, MORELLO op.cit. (nota 4), p.199; COLLARETA, Cristalli op. cit. (nota 1) p. 505; CASELLI L'ornamento op. cit. (nota 1) p. 89-90; FOBELLI, op. cit. (nota 9). Per la croce di San Candido: HAHNLOSER, BRUGGER KOCH, op. cit. (nota 1), n. 145, p. 128; DEGEN op. cit. (nota 1), pp. 274-288; F. KIRCHEWEGER, scheda 83, in FILLITZ, MORELLO op.cit. (nota 4), p.200; CASELLI L'ornamento op. cit. (nota 1) pp. 97-98.

${ }^{45}$ Per la croce di Udine: HAHNLOSER, BRUGGER KOCH, op. cit. (nota 1), n. 111, p. 116; DEGEN op. cit. (nota 1), pp. 319-325; C. GABERSCHEK, scheda 116, in FILLITZ, MORELLO op.cit. (nota 4), pp.242-243.

${ }^{46}$ Per la croce di Foligno: HAHNLOSER, BRUGGER KOCH, op. cit. (nota 1), n. 146, p. 128; DEGEN op. cit. (nota 1), pp. 270-278; COLLARETA, Cristalli op. cit. (nota 1); SANTANICCHIA, op. cit. (nota 1).

${ }^{47}$ HAHNLOSER, BRUGGER KOCH, op. cit. (nota 1), n. 106, p. 114; DEGEN op. cit. (nota 1), pp. 355-356.

${ }^{48}$ C. BERTELLI, La croce di Chiaravalle, in Il re dei confessori, dalla croce dei Cloisters alle croci italiane, Milano 1984, pp.121- 131; L. CASELLI, La croce di Chiaravalle milanese e le croci veneziane in cristallo di rocca, Padova 2002; COLLARETA, Il cristallo op. cit. (nota 1), pp. 502-505. 


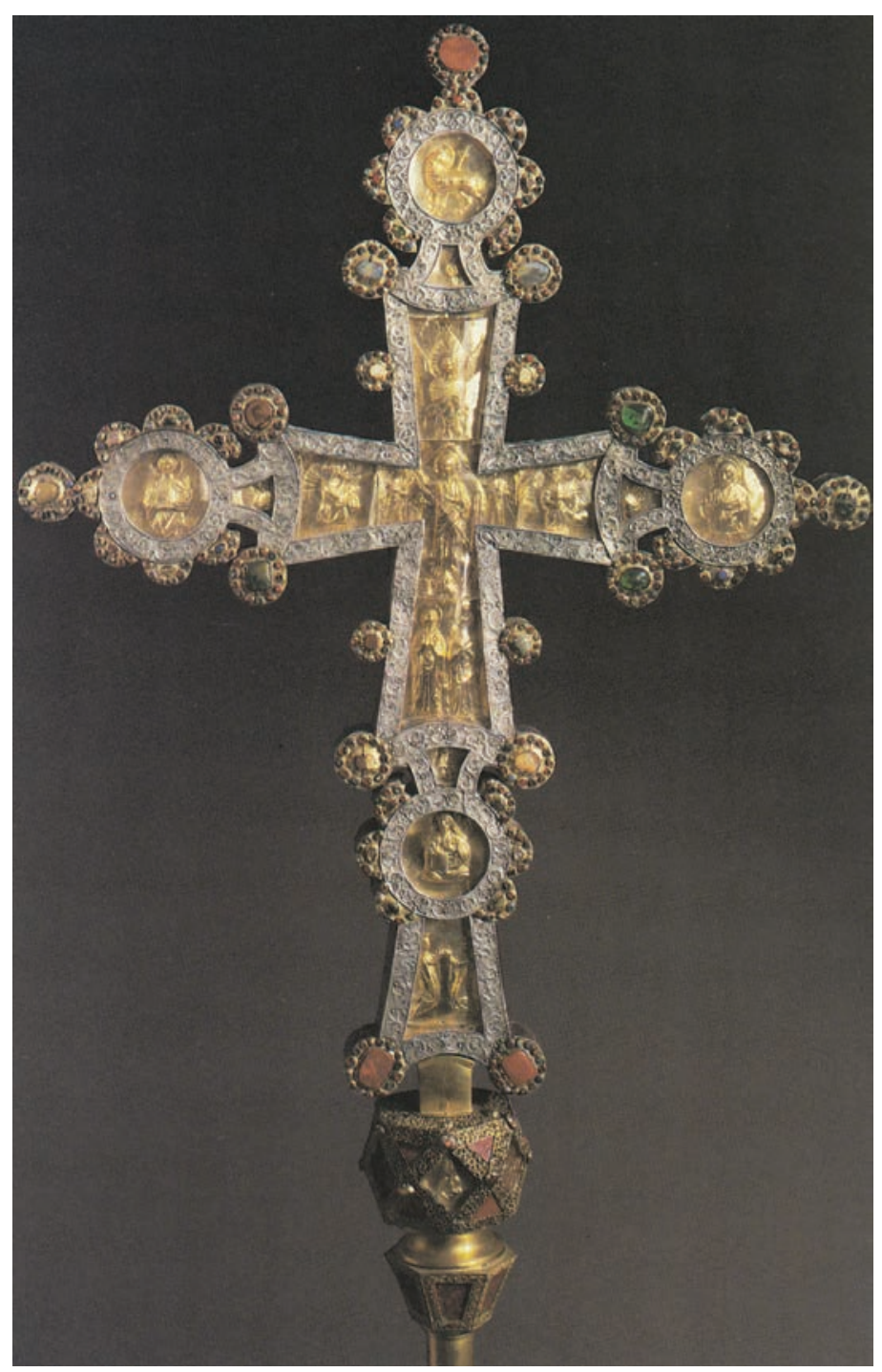

Fig. 16. Milano, Tesoro del Duomo, Croce di Chiaravalle (foto da CASELLI, 2002, p. 128).

rappresentano la crocifissione con i dolenti , Costantino ed Elena e il Precursore, (Fig. 3) mentre sul verso è rappresentata la Parusia a sbalzo d'argento sotto cristallo. (Fig. 16)
I materiali preziosi utilizzati per questo particolare genere hanno una valenza simbolica: il cristallo infatti con la sua trasparenza simboleggia la purezza della luce e quindi la manifestazione di Dio, mentre il diaspro per il suo colore è associato al Sangue di Cristo. ${ }^{49}$ La scelta quindi di accostare questi materiali, attestata in ambito profano fin dalle descrizioni degli oggetti portati nel 1263 da Tommaso Vilio in Persia, ${ }^{\circ}$ acquista particolari valenze nell'impiego sacro di questi contenitori, poiché in gran parte si tratta di croci santificate dalla reliquia più importante per la cristianità, quella del legno dello strumento del martirio di Cristo.

A questo punto è necessario porre un problema: l'opera dei cristalleri è in molti casi veicolo e contenitore di reliquie, destinate, come riscontriamo dalle collocazioni degli oggetti e dalle notizie storiche ad essi relative, all'esportazione. La reliquia era per i committenti e acquirenti ben più preziosa dell'oggetto che la mostrava e/o conteneva che - nella sua preziosità materiale - esaltava il contenuto e rispettava $\mathrm{i}$ dettami biblici. Quindi, chi metteva a disposizione le reliquie? Sappiamo che con il sacco costantinopolitano arrivò nel Tesoro di San Marco una reliquia della croce, cui si aggiunsero successivamente altri reliquiari della Passione, ${ }^{51} \mathrm{ma}$ dobbiamo interrogarci sulla provenienza dei frammenti del Legno della Croce montati nelle croci di cristallo. Nel corso del XIII secolo c'è un grande circolazione di reliquie, sappiamo che a Venezia sono presenti reliquiari anche in mano privata (reliquiario a San Samuele, un reliquiario Morosini arrivato da Costantinopoli e donato poi a Santa Corona di Vicenza $)^{52}$ e possiamo immaginare quindi una sorta di commercio di reliquie, veicolato anche attraverso queste croci, questi dittici, questi altaroli. Se per alcune croci reliquiario possiamo ipotizzare una committenza privata e acquisti ad hoc sulla piazza veneziana, ${ }^{33}$ possiamo anche prospettare la possibilità che in qualche caso si sia trattato di doni preziosi finalizzati a rapporti diplomatici? ${ }^{54}$

Per i supporti i produttori veneziani dispongono di materiali giunti sulla piazza di Venezia attraverso molteplici vie, marittime ma anche terrestri. ${ }^{55}$

Le norme del capitolare - come ho già ricordato - riguardano anche le modalità di acquisto delle partite di cristalli,

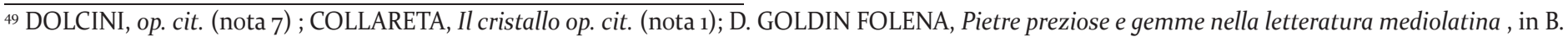
ZANETTIN ( a cura di ), Cristalli e gemme, realtà fisica e immaginario, simbologia, tecniche e arte, Venezia 2003, pp. 599- 622; SANTANICCHIA, op. cit. (nota 1), pp. 122-124.

$5^{50}$ Vedi note 24-25.

${ }^{51}$ Dandolo (ed. PASTORELLO op. cit. (nota 39), p. 280) riporta l'elenco delle reliquie ottenute nel 1204 con la spartizione del bottino costantinopolitano: una reliquia della Vera Croce, il braccio di san Giorgio, la testa di san Giovanni, Preziosissimo sangue di Cristo. Nel Tesoro di San Marco sono presenti più reliquiari della Croce: quello giunto nel 1204 è con molta probabilità il reliquiario bizantino in tavola d'argento con le immagini di Costantino ed Elena, rimontato nel XVI secolo, STEINGRABER scheda 192 in HAHNLOSER op. cit. (nota 4), pp. 191-193. H. HELLENKEMPER, I trofei del doge, in Tesoro op. cit 1986 (nota 4), pp. 31-41; R.POLACCO, Proposte per una chiarificazione sul significato e sulla funzione del bassorilievo delle reliquie dell'andito Foscari in San Marco a Venezia, in E. CONCINA, G. TROVABENE, M. AGAZZI (a cura di), Hadriatica, attorno a Venezia e al medioevo tra arti, storia e storiografia ; scritti in onore di Wladimiro Dorigo, Padova, 2002, pp. 133-137; H. A. KLEIN, Die Heiltümer von Venedig, die byzantinischen Reliquien der Stadt , in G. ORTALLI, G. RAVEGNANI, P. SCHREINER ( a cura di), Quarta crociata, Venezia, Bisanzio, Impero latino, Venezia, 2006,vol.II, pp. 789- 823: 798-803.

${ }^{52}$ Un reliquiario bizantino della Croce è donato da Marco Venier all'inizio del XV secolo alla chiesa di San Samuele di Venezia, ora in Santo Stefano ( G. ROSSI SCARPA, scheda n.14, in Tesori della fede, Venezia 2000, p. 163; S. PIGHI, scheda 71 in CAPUTO, GENTILI, op. cit. (nota 7), pp.176-177, fig p. 123) ed è collegabile a quello pervenuto alla collezione Stoclet, Bruxelles (Collection Adolphe Stoclet: Choix d'oeuvre appartenant a Madame Feron-Stoclet, Bruxelles 1957, pp. 158-161; A. CUTLER, From Loot to Scholarship: Changing Modes in the Italian Response to Byzantine Artifacts ca. 1200-1750, in Dumbarton Oaks Paper, 49, 1995, pp. 237 - 267: p.241). Una icona - reliquiario della croce e di santi (con teche sotto cristalli) è donata da Filippa, vedova di un Marino Morosini, al vescovo Bartolomeo da Breganze fondatore della chiesa di Santa Corona a Vicenza ed è certificata la sua provenienza da Costantinopoli con una autentica del 1243 ( F. LOMASTRO TOGNATO ( a cura di ), I «Monumenta reliquiarum » di S. Corona di Vicenza, Padova 1992, pp. 16-17,38).

53 Per la circolazione in centro Italia dei prodotti dei cristalleri vedi FOBELLI op. cit. (nota 9) e SANTANICCHIA op. cit. (nota 1).

${ }^{54}$ H. BELTING, Introduction, in H. BELTING (a cura di), Il Medio Oriente e l'Occidente nell'arte del 13. secolo ,Bologna 1982, pp. 1 -10; CUTLER, op. cit. (nota 52). ${ }_{55}$ Diaspro e cristallo dovevano provenire infatti dall'Europa: DOLCINI, op. cit. (nota 7). Per la circolazione di merci lungo le direttrici alpine (via d'Alemagna in primis) si veda RIEDMANN, op. cit. (nota 29) e HARTEL, op. cit. (nota 31), pp. 589-590. 
destinati all'arte "grossa” e "minuta" (differenziazione legata alle dimensioni dei cristalli e ai diversi prodotti ricavabili): questi dovevano avvenire sulla piazza veneziana (ma non esclusivamente), approvvigionata anche da mercanti stranieri..$^{56} \mathrm{Il}$ cristallo molto probabilmente era cristallo europeo, proveniente da nord ( dalla Svizzera o dai Carpazi) ${ }^{57}$ di cui dovevano essere tramite i tedeschi, attivi a Venezia fin dal XII secolo con base nel Fondaco loro destinato dal 1222 nella zona di San Bartolomio dove risiedevano e lavoravano orefici in gran numero..$^{8}$

A Venezia quindi nel XIII secolo, durante la fase 'imperiale' dopo la conquista di Costantinopoli e nell'ambito degli scambi culturali non solo tra Oriente e Occidente, ma anche tra gli occidentali conquistatori, matura una nuova esperienza creativa che elabora nuovi oggetti, frutto della commistione di tecniche e materiali disponibili sulla piazza veneziana grazie alla funzione portuale e di interconnessione di reti commerciali terrestri e marittime. Sono oggetti destinati per la maggior parte al mercato straniero: nessuna croce o tavola con miniature sotto cristallo e diaspro si conserva a Venezia; i destinatari sono riferibili a un pubblico elevato e non si può escludere che in qualche caso si sia trattato di doni diplomatici, ma dobbiamo porci anche il problema degli investitori. Nel testamento di Vilio del 1263 gli oggetti di cristallo in viaggio per essere venduti sono comproprietà di più persone (il padre Vitale Vilio, un Minio, un Dandolo): si tratta evidentemente di un investimento su beni di lusso di alta rendita che al rientro del mercante viaggiatore avrebbero fatto realizzare il guadagno. 59 In questo caso si trattava quindi di prodotti i cui committenti erano investitori, non fruitori degli oggetti.

Nella grande fucina di idee e di forme della Venezia duecentesca, che fin dalla prima metà del secolo era divenuta centro di riferimento per la produzione orafa (come prova il documento legato alla realizzazione di «çogie» per l'imperatore Federico II nel 1225$)^{60}$ si creano quindi - per un vasto mercato - miniature sotto cristallo a imitazione di smalti bizantini, ${ }^{61}$ croci sfavillanti, oggetti di devozione, di arredo liturgico, ma anche profani, montati e decorati con elementi a sbalzo e/o con la filigrana, un'altra componente essenziale del polimaterismo dei cristalleri. Anche per la filigrana possiamo parlare di importazione, ma essa diverrà totalmente veneziana, tanto che il nome opus ad filum viene mutato in opus veneticum:62 l'ennesima fagocitazione dei veneziani. Successivamente - nel XIV secolo - accanto alle miniature si adotterà un' altra tecnica innovativa - lo smalto traslucido - immessa nel Tesoro di San Marco con la realizzazione del reliquiario del braccio di San Giorgio e il nuovo piede di un calice bizantino in serpentino. ${ }^{63}$ Per tutto il XIV secolo si varieranno e si modificheranno singole componenti, ma gli schemi principali resteranno quelli impostati nel Duecento da personalità che non possiamo individuare, dotate di grande abilità nel mixare e rifunzionalizzare i materiali, rispondendo alla richiesta di un bacino europeo che trovava a Venezia un centro di riferimento per una innovativa declinazione dell'arte orafa, in sostituzione del ruolo avuto da Costantinopoli fino alla sua conquista. ${ }^{64}$

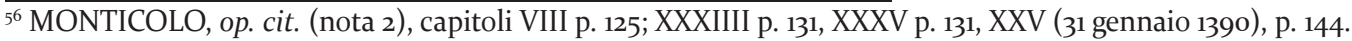

${ }^{57}$ DOLCINI, op. cit. (nota 7) pp. 345- 346; LAIOU, op. cit. (nota 9).

${ }^{58}$ DORIGO, op. cit. (nota 15), p 742. Dal 1319 tutti gli orefici furono obbligati a risiedere a Rialto.

59 Nello stesso testamento è indicato il valore degli oggetti più preziosi: vedi nota 25.

${ }^{60}$ Il documento (pubblicato da MONTICOLO, op. cit. 1896 (nota 3), p. 257) è l'obbligazione di Vitale Nadal che si impegna a consegnare entro un anno in Puglia i gioielli realizzati con le pietre preziose dell'imperatore Federico II consegnate al padre Marino Nadal.

${ }^{61}$ Un altro prodotto sono le miniature sotto cristallo cucite in abiti e accessori: si veda la Mitra di Traù: D. DAVANZO POLI, Pietro e Marco nei ricami medievali, in CASELLI L. (a cura di), San Pietro e San Marco arte e iconografia in area adriatica, Roma 2009, pp.185-203; SPIANDORE, op. cit. (nota 1).

${ }^{62}$ I. HUECK, De opere duplici venetico, in Mitteilungen der Kunsthistorische Institute in Florenz, XII, 1965, pp. 1-30;D. GABORIT CHOPIN, La filigrana veneziana, in Tesoro 1986 op. cit. (nota 4), pp. 241-244; L. CASELLI, Opus veneticum ad filum et opus duplex: considerazioni su alcune opere di oreficeria veneziana del Duecento, in L. CASELLI - J. SCARPA - G.TROVABENE (a cura di), Venezia : arti e storia : studi in onore di Renato Polacco, Venezia [2005], pp. 53 - 6o. ${ }^{63}$ E' il caso della croce di Assisi (vedi nota 43 ). Per il reliquiario di San Giorgio e il cd Calice di Serpentino: HAHNLOSER, op. cit. (nota 4), nn. 159 e 61; W. DIXOM, scheda 41, in Tesoro 1986 op. cit (nota 4), pp. 290-293; D. ALCOUFFE, D. GABORIT CHOPIN, scheda 42 in Tesoro 1986 op. cit (nota 4), pp. $294-299$. Per lo smalto a traslucido : G. MARIANI CANOVA op. cit e SPIANDORE op. cit. (nota 43).
}

${ }^{64}$ LAIOU, op. cit. (nota 9). 